\title{
Republic of Estonia: Statistical Appendix
}

This Statistical Appendix paper on the Republic of Estonia was prepared by a staff team of the International Monetary Fund as background documentation for the periodic consultation with the member country. It is based on the information available at the time it was completed on June 14, 2002. The views expressed in this document are those of the staff team and do not necessarily reflect the views of the government of the Republic of Estonia or the Executive Board of the IMF.

The policy of publication of staff reports and other documents by the IMF allows for the deletion of market-sensitive information.

To assist the IMF in evaluating the publication policy, reader comments are invited and may be sent by e-mail to Publicationpolicy@imf.org.

Copies of this report are available to the public from

International Monetary Fund - Publication Services

$70019^{\text {th }}$ Street, N.W. - Washington, D.C. 20431

Telephone: (202) 623-7430 - Telefax: (202) 623-7201

E-mail: publications@imf.org Internet: http://www.imf.org

Price: $\$ 15.00$ a copy

\section{International Monetary Fund Washington, D.C.}




\title{
INTERNATIONAL MONETARY FUND
}

\author{
REPUBLIC OF ESTONIA
}

\section{Statistical Appendix}

Prepared by Robert Burgess, Alfred Schipke, Niamh Sheridan, Wayne Mitchell and Gohar Abajyan

Approved by European II Department

June 14,2002

Contents

Basic Data 3

Statistical Appendix Tables

1. Gross Domestic Product by Expenditure, 1997-2001 …........................................... 4

2. Gross Domestic Product by Origin, 1997-2001 ……............................................. 5

3. Real Gross Domestic Product by Origin, 1997-2001 ............................................. 6

4. Gross Domestic Product by Income Approach, 1997-2001 ..................................... 7

5. Prices, 1997-2002 ........................................................................................ 8

6. Average Monthly Wage, 1997-2001 …………................................................. 9

7. Average Monthly Wage by Sector, 1997-2001 .................................................. 10

8. Employment by Sector, 1997-2001 ……........................................................... 11

9. Labor Market Indicators, 1997-2002 ............................................................. 12

10. Unit Labor Costs by Sector, 1997-2001 ……...................................................... 13

11. Summary of General Government Operations, 1997-2002 ..................................... 14

12. General Government Revenue, 1997-2002 ……............................................... 15

13. General Government Expenditure, 1997-2002 …............................................... 16

14. Fiscal Balances by Government Sector (In million of kroons), 1997-2001 ............. 17

15. Fiscal Balances by Government Sector (In percent of GDP), 1997-2001 ................ 18

16. Banking Survey and Monetary Authorities, 1997-2002 .......................................... 19

17. Maturity and Currency Composition of Deposits, 1997-2002 …........................... 20

18. Maturity and Currency Composition of Loans, 1997-2002 .................................... 21

19. Average Interest Rates of Deposits and Loans, 1997-2002 .................................... 22

20. Nonperforming Loans of Commercial Banks, 1997-2002 …................................ 23

21. Commercial Bank Profits (on a consolidated basis), 1997-2002 …....................... 24

22. Balance of Payments, 1997-2001 …………...................................................... 25

23. Direction of Trade - Exports by Countries, 1997-2001 ………………………….... 26

24. Direction of Trade - Imports by Countries, 1997-2001 ....................................... 27

25. Composition of Trade - Exports by Commodities, 1997-2001............................... 28 
26. Composition of Trade - Imports by Commodities, 1997-2001 ............................... 29

27. Foreign Direct Investment Inflows by Countries, 1997-2001 .............................. 30

28. Foreign Direct Investment Outflows by Countries, 1997-2001.............................. 30

29. Foreign Direct Investment Inflows by Sectors, 1997-2001 .................................. 31

$30 \quad$ Foreign Direct Investment Outflows by Sectors, 1997-2001 .................................. 31

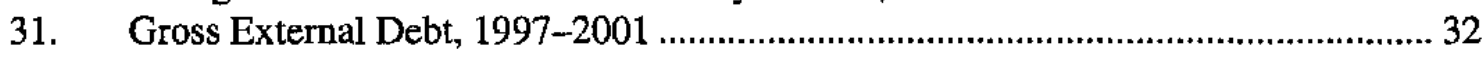

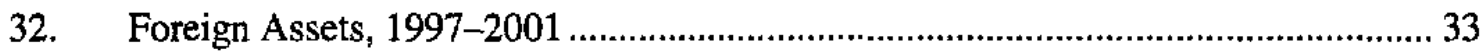

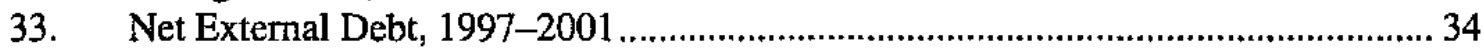


Estonia: Basic Data

Social and demographic indicators, 2000

Area

Population

Rate of population grow'th

Life expectancy at birh $\mathbf{l}$

Male

Female

Infant mortaiity rate (per 1,000 births) $1 /$

Hospital beds (per 10,000 inhabitants) I/

Physicians (per 10,000 inhabitants) I/
45,227 sç. km.

1.3642 million

-0.4 percent per year

70.6 years

65.3 years

76.1 years

9.5

71.9

39.9

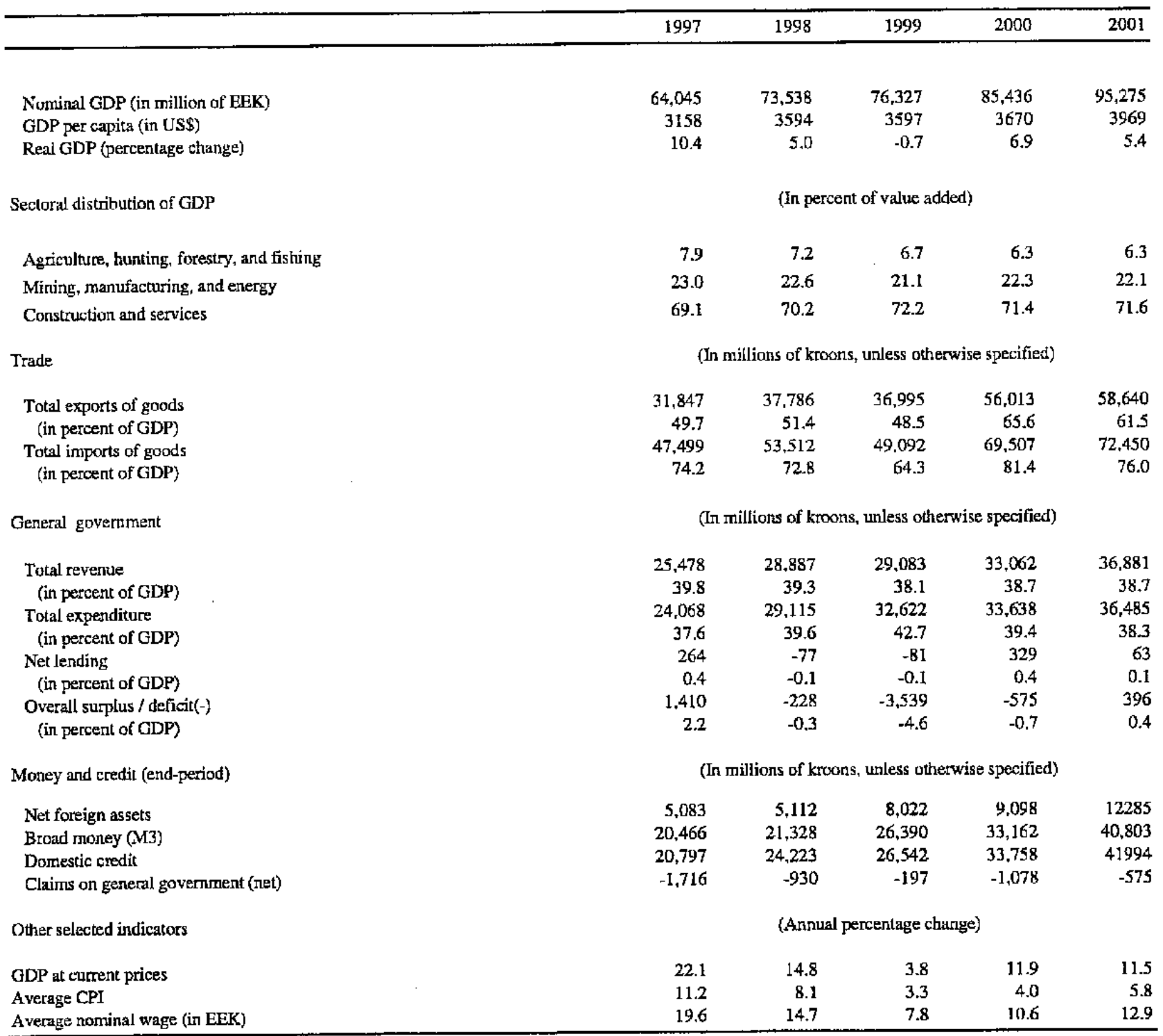

Sources: Estonian authorities; and Fund staff estimates.

1/ Data for 1999. 
Table 1. Estonia: Gross Domestic Product by Expenditure, 1997-2001

\begin{tabular}{|c|c|c|c|c|c|}
\hline & 1997 & 1998 & 1999 & 2000 & 2001 \\
\hline & \multicolumn{5}{|c|}{ (In million kroons, current prices) } \\
\hline Consumption & 52,689 & 59,999 & 62,276 & 68,467 & 74,256 \\
\hline Private consumption & 37,586 & 42,927 & 43,922 & 49,299 & 53,913 \\
\hline General government consumption & 14,728 & 16,651 & 17,851 & 18,594 & 19,720 \\
\hline Non-profit institutions consumption & 375 & 421 & 503 & 574 & 623 \\
\hline Investment & 19,845 & 21,572 & 18,715 & 21,037 & 25,426 \\
\hline Gross fixed capital formation & 17,962 & 21,761 & 19,023 & 19,965 & 24,212 \\
\hline Changes in inventories & 1,882 & -189 & -308 & 1,072 & 1,213 \\
\hline Domestic demand & 72,534 & 81,571 & 80,990 & 89,504 & 99,682 \\
\hline Total exports (fob) & 50,213 & 58,590 & 58,947 & 81,499 & 87,432 \\
\hline Exports of goods (fob) & 31,847 & 37,786 & 36,995 & 56,013 & 58,640 \\
\hline Exports of services (fob) & 18,367 & 20,804 & 21,952 & 25,486 & 28,792 \\
\hline Total imports (fob) & 57,633 & 66,267 & 62,703 & 85,802 & 91,403 \\
\hline Imports of goods (fob) & 47,499 & 53,512 & 49,092 & 69,507 & 72,450 \\
\hline Imports of services (fob) & 10,134 & 12,755 & 13,610 & 16,296 & 18,954 \\
\hline Statistical Discrepancy & $-1,069$ & -356 & -908 & 236 & -436 \\
\hline \multirow[t]{2}{*}{ GDP } & 64,045 & 73,538 & 76,327 & 85,436 & 95,275 \\
\hline & \multicolumn{5}{|c|}{ (In million kroons, constant 1995 prices) } \\
\hline Consumption & 38,991 & 40,991 & 41,231 & 43,664 & 44,729 \\
\hline Private consumption & 27,951 & 29,456 & 29,227 & 31,622 & 32,641 \\
\hline General government consumption & 10,766 & 11,250 & 11,675 & 11,682 & 11,718 \\
\hline Non-profit institutions consumption & 274 & 285 & 329 & 361 & 370 \\
\hline Investment & 15,359 & 15,260 & 12,926 & 14,151 & 16,538 \\
\hline Gross fixed capital formation & 13,836 & 15,404 & 13,155 & 13,413 & 15,722 \\
\hline Changes in inventories & 1,523 & -143 & -229 & 738 & 817 \\
\hline Domestic demand & 54,350 & 56,251 & 54,157 & 57,815 & 61,267 \\
\hline Total exports (fob) & 39,224 & 44,158 & 43,901 & 57,373 & 56,233 \\
\hline Exports of goods (fob) & 26,583 & 30,922 & 30,125 & 42,135 & 39,636 \\
\hline Exports of scrvices (fob) & 12,641 & 13,236 & 13,776 & 15,237 & 16,597 \\
\hline Total imports (fob) & 45,654 & 51,396 & 48,433 & 62,489 & 62,782 \\
\hline Imports of goods (fob) & 38,661 & 42,764 & 39,377 & 52,204 & 52,080 \\
\hline Imports of services (fob) & 6,993 & 8,632 & 9,056 & 10,284 & 10,702 \\
\hline Statistical Discrepancy & -951 & 326 & -629 & -330 & 474 \\
\hline GDP & 46,969 & 49,339 & 48,996 & 52,369 & 55,192 \\
\hline
\end{tabular}

Source: National authorities. 
Table 2. Estonia: Gross Domestic Product by Origin, 1997-2001

\begin{tabular}{|c|c|c|c|c|c|}
\hline & 1997 & 1998 & 1999 & 2000 & 2001 \\
\hline & \multicolumn{5}{|c|}{ (In million kroons, current prices) } \\
\hline Agriculture and hunting & 2,780 & 2,810 & 2,584 & 2.784 & 3,186 \\
\hline Forestry & 1,347 & 1,614 & 1,812 & 1,843 & 1,937 \\
\hline Fishing & 340 & 327 & 235 & 203 & 219 \\
\hline Mining and quanrying & 830 & 796 & 754 & 857 & 915 \\
\hline Manufacturing & 10,193 & 11,709 & 11,355 & 13,644 & 15,222 \\
\hline Electricity, gas and water supply & 1,984 & 2,411 & 2,464 & 2,524 & 2,712 \\
\hline Construction & 3,538 & 4,425 & 4,121 & 4,461 & 4,938 \\
\hline Services & 35,522 & 41,955 & 45,687 & 49,975 & 56,047 \\
\hline Wholesale and retail trade & 8,539 & 9,871 & 9,945 & 11,090 & 12,403 \\
\hline Hotels and restaurants & 714 & 843 & 939 & 1,000 & 1,131 \\
\hline Transport, storage and communications & 7,248 & 9,427 & 10,479 & 11,735 & 13,816 \\
\hline Real estate, renting and business activities & 5,882 & 7,317 & 7,738 & 8,462 & 9,527 \\
\hline Financial intermediation & 2,366 & 2,398 & 2,777 & 3,202 & 3,693 \\
\hline Public administration & 2,597 & 2,900 & 3,506 & 3,692 & 3,947 \\
\hline Education & 3,121 & 3,637 & 4,204 & 4,277 & 4,608 \\
\hline Health and social work & 2,231 & 2,415 & 2,783 & 2,949 & 3,004 \\
\hline Other services & 2,824 & 3,147 & 3,315 & 3,568 & 3,917 \\
\hline Total value added & 56,533 & 66,048 & 69,011 & 76,290 & 85,175 \\
\hline FISIM $1 /$ & 1,196 & 962 & 1,093 & 1,250 & 1,450 \\
\hline GDP at basic prices & 55,338 & 65,086 & 67,919 & 75,040 & 83,724 \\
\hline Net taxes on product & 8,707 & 8,452 & 8,409 & 10,396 & 11,551 \\
\hline \multirow[t]{2}{*}{ GDP at market prices } & 64,045 & 73,538 & 76,327 & 85,436 & 95,275 \\
\hline & \multicolumn{5}{|c|}{ (In percent of GDP) } \\
\hline Agriculture and hunting & 4.3 & 3.8 & 3.4 & 3.3 & 3.3 \\
\hline Forestry & 2.1 & 2.2 & 2.4 & 2.2 & 2.0 \\
\hline Fishing & 0.5 & 0.4 & 0.3 & 0.2 & 0.2 \\
\hline Mining and quarrying & 1.3 & 1.1 & 1.0 & 1.0 & 1.0 \\
\hline Manufacturing & 15.9 & 15.9 & 14.9 & 16.0 & 16.0 \\
\hline Electricity, gas and water supply & 3.1 & 3.3 & 3.2 & 3.0 & 2.8 \\
\hline Construction & 5.5 & 6.0 & 5.4 & 5.2 & 5.2 \\
\hline Services & 55.5 & 57.1 & 59.9 & 58.5 & 58.8 \\
\hline Wholesale and retail trade & 13.3 & 13.4 & 13.0 & 13.0 & 13.0 \\
\hline Hotels and restaurants & 1.1 & 1.1 & 1.2 & 1.2 & 1.2 \\
\hline Transport, storage and communications & 11.3 & 12.8 & 13.7 & 13.7 & 14.5 \\
\hline Real estate, renting and business activities & 9.2 & 10.0 & 10.1 & 9.9 & 10.0 \\
\hline Financial intermediation & 3.7 & 3.3 & 3.6 & 3.7 & 3.9 \\
\hline Public administration & 4.1 & 3.9 & 4.6 & 4.3 & 4.1 \\
\hline Education & 4.9 & 4.9 & 5.5 & 5.0 & 4.8 \\
\hline Health and sociai work & 3.5 & 3.3 & 3.6 & 3.5 & 3.2 \\
\hline Other services & 4.4 & 4.3 & 4.3 & 4.2 & 4.1 \\
\hline Total value added & 88.3 & 89.8 & 90.4 & 89.3 & 89.4 \\
\hline FISIM $1 /$ & 1.9 & 1.3 & 1.4 & 1.5 & 1.5 \\
\hline GDP at basic prices & 86,4 & 88.5 & 89.0 & 87.8 & 87.9 \\
\hline Net taxes on product & 13.6 & 11.5 & 11.0 & 12.2 & 12.1 \\
\hline GDP at market prices & 100.0 & 100.0 & 100.0 & 100.0 & 100.0 \\
\hline
\end{tabular}

Source: National authorities.

1/ Financial intermediation services indirectly measured. 
Table 3. Estonia: Real Gross Domestic Product by Origin, 1997-2001

\begin{tabular}{|c|c|c|c|c|c|}
\hline & 1997 & 1998 & 1999 & 2000 & 2001 \\
\hline & \multicolumn{5}{|c|}{ (In million kroons, constant 1995 prices) } \\
\hline Agriculture and hunting & 2,025 & 1,930 & 1,779 & 1,748 & 1,842 \\
\hline Forestry & 1,046 & 1,131 & 1,235 & 1,216 & 1,238 \\
\hline Fishing & 298 & 287 & 220 & 181 & 190 \\
\hline Mining and quarrying & 733 & 681 & 610 & 661 & 701 \\
\hline Manufacturing & 8,328 & 8,850 & 8,765 & 10,151 & 10,954 \\
\hline Electricity, gas and water supply & 1,443 & 1,328 & 1,230 & 1,231 & 1,235 \\
\hline Construction & 2,898 & 3,467 & 3,164 & 3,376 & 3,562 \\
\hline Services & 25,162 & 26,492 & 27,089 & 28,530 & 29,956 \\
\hline Wholesale and retail trade & 6,062 & 6,408 & 6,428 & 6.921 & 7,373 \\
\hline Hotels and restaurants & 538 & 612 & 679 & 709 & 771 \\
\hline Transport, storage and communications & 5,084 & 5,579 & 5,876 & 6,310 & 6,741 \\
\hline Real estate, renting and business activities & 4,217 & 4,715 & 4,715 & 4,890 & 5,159 \\
\hline Financial intermediation & 1,663 & 1,504 & 1,626 & 1,779 & 1,938 \\
\hline Public administration & 1,872 & 1,883 & 1,899 & 1,921 & 1,937 \\
\hline Education & 2,285 & 2,315 & 2,313 & 2,309 & 2,334 \\
\hline Health and social work & 1,546 & 1,601 & 1,647 & 1,693 & 1,646 \\
\hline Other services & 1,896 & 1,875 & 1,906 & 1,999 & 2,056 \\
\hline Total value added & 41,931 & 44,166 & 44,091 & 47,094 & 49,676 \\
\hline FISIM $1 /$ & 874 & 650 & 715 & 786 & 862 \\
\hline GDP at basic prices & 41,057 & 43,517 & 43,377 & 46,309 & 48,814 \\
\hline Net taxes on product & 5,912 & 5,823 & 5,619 & 6,060 & 6,378 \\
\hline \multirow{2}{*}{ GDP at market prices } & 46,969 & 49,339 & 48,996 & 52,369 & 55,192 \\
\hline & \multicolumn{5}{|c|}{ (year-on-year growth rates in percent) } \\
\hline Agriculture and hunting & -2.6 & -4.7 & -7.8 & -1.7 & 5.3 \\
\hline Forestry & 23.0 & 8.2 & 9.2 & -15 & 1.8 \\
\hline Fishing & 26.3 & -3.5 & -23.3 & -18.0 & 5.0 \\
\hline Mining and quarrying & 13.4 & -7.1 & -10.5 & 8.5 & 6.0 \\
\hline Manufacturing & 16.9 & 6.3 & -1.0 & 15.8 & 7.9 \\
\hline Electricity, gas and water supply & .2 .2 & -8.0 & -7.4 & 0.1 & 0.3 \\
\hline Construction & 15.7 & 19.7 & -8.7 & 6.7 & 5.5 \\
\hline Services & 8.5 & 5.3 & 2.3 & 5.3 & 5.0 \\
\hline Wholesale and retail trade & 3.2 & 5.7 & 0.3 & 7.7 & 6.5 \\
\hline Hotels and restaurants & 6.6 & 13.8 & 10.9 & 4.4 & 8.8 \\
\hline Transport, storage and communications & 17.8 & 9.7 & 5.3 & 7.4 & 6.8 \\
\hline Real estate, renting and business activities & 12.4 & 11.8 & 0.0 & 3.7 & 5.5 \\
\hline Financial intermediation & 16.4 & -9.6 & 8.2 & 9.4 & 9.0 \\
\hline Public administration & 5.4 & 0.6 & 0.8 & 1.2 & 0.8 \\
\hline Education & 0.8 & 1.3 & -0.1 & -0.2 & 1.1 \\
\hline Health and social work & 4.0 & 3.6 & 2.8 & 2.8 & -2.8 \\
\hline Other services & 5.7 & -1.1 & 1.7 & 4.8 & 2.9 \\
\hline Total value added & 10.0 & 5.3 & -0.2 & 6.8 & 5.5 \\
\hline FISIM $1 /$ & 11.0 & -25.7 & 10.0 & 10.0 & 9.7 \\
\hline GDP at basic prices & 10.0 & 6.0 & -0.3 & 6.8 & 5.4 \\
\hline Net taxes on product & 13.6 & -1.5 & -3.5 & 7.9 & 5.2 \\
\hline GDP at market prices & 10.4 & 5.0 & -0.7 & 6.9 & 5.4 \\
\hline
\end{tabular}

Source: National authorities.

1/ Financial intermediation services indirectly measured. 
$-7-$

Table 4. Estonia: Gross Domestic Product by Income Approach, 1997-2001

\begin{tabular}{|c|c|c|c|c|c|}
\hline & 1997 & 1998 & 1999 & 2000 & 2001 \\
\hline & \multicolumn{5}{|c|}{ (In millions of kroons, current prices) } \\
\hline Compensation of employees & 33,402 & 37,478 & 41,049 & 42,334 & 46,509 \\
\hline Wages and salaries & 25,433 & 28,433 & 31,096 & 32,049 & 35,181 \\
\hline Employer's social contributions & 7,968 & 9,045 & 9,953 & 10,285 & 11,328 \\
\hline Consumption of fixed capital & 7,511 & 10,111 & 11,429 & 12,397 & 14,123 \\
\hline Taxes on production and imports & 10,006 & 10,034 & 9,966 & 12,123 & 13,476 \\
\hline Subsidies & 458 & 805 & 899 & 852 & 963 \\
\hline Operating surplus and mixed income 1 / & 13,584 & 16,720 & 14,783 & 19,434 & 22,131 \\
\hline GDP at market prices & 64,045 & 73,538 & 76,327 & 85,436 & 95,275 \\
\hline
\end{tabular}

Source: National authorities.

1/ Does not include financial intermediation services indirectly measured. 
Table 5. Estonia: Prices, 1997-2002

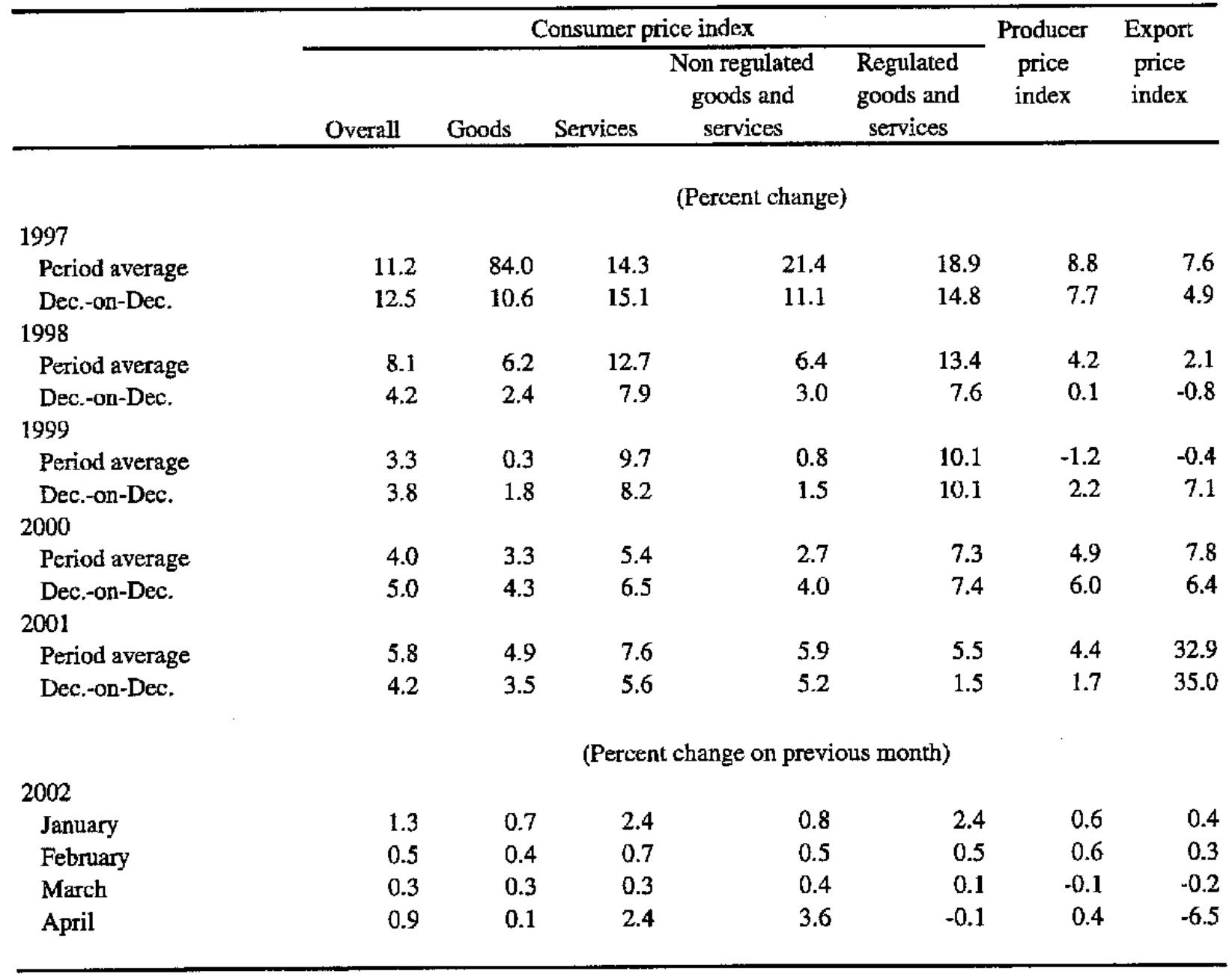

Source: National authorities. 
Table 6. Estonia: Average Monthly Wage, 1997-2001

\begin{tabular}{|c|c|c|c|}
\hline & $\begin{array}{c}\text { Nominal wage in } \\
\text { kroons }\end{array}$ & $\begin{array}{c}\text { Real wage } \\
(1992=100) 1 /\end{array}$ & $\begin{array}{c}\text { Nominal wage in } \\
\text { US dollars }\end{array}$ \\
\hline 199721 & 3,571 & 130 & 257 \\
\hline $19982 /$ & 4,096 & 138 & 292 \\
\hline $19992 /$ & 4,416 & 145 & 301 \\
\hline $20002 /$ & 4,883 & 154 & 288 \\
\hline $20012 /$ & 5,514 & 164 & 314 \\
\hline \multicolumn{4}{|l|}{2000} \\
\hline March & 4,598 & 147 & 284 \\
\hline June 3/ & 5,548 & 176 & 337 \\
\hline September & 4,791 & 150 & 267 \\
\hline December $3 /$ & 5,740 & 177 & 329 \\
\hline \multicolumn{4}{|l|}{2001} \\
\hline March & 5,227 & 158 & 298 \\
\hline June $3 /$ & 6,206 & 184 & 341 \\
\hline September & 5,376 & 159 & 313 \\
\hline December 3/ & 6,345 & 187 & 362 \\
\hline
\end{tabular}

Source: National authorities.

1/ Nominal wage deflated by CPI.

2/ Annual average calculated as the arithmetic mean of monthly average wages.

3/ Includes mid-year and end-year bonus payments. 
Table 7. Estonia: Average Monthly Wage by Sector, 1997-2001 1/

\begin{tabular}{lrrrrr}
\hline & 1997 & 1998 & 1999 & 2000 & 2001 \\
\hline & \multicolumn{5}{c}{ (In kroons) } \\
Total 2l & 3,573 & 4,125 & 4,440 & 4,876 & 5,511 \\
& & & & & \\
Agriculture and hunting & 2,131 & 2,535 & 2,385 & 2,788 & 3,241 \\
Forestry & 3,657 & 4,059 & 4,134 & 4,329 & 4,915 \\
Fishing & 3,640 & 3,674 & 3,496 & 3,578 & 3,778 \\
Mining and quarrying & 4,412 & 4,894 & 5,152 & 5,889 & 6,859 \\
Manufacturing & 3,578 & 4,081 & 4,117 & 4,769 & 5,157 \\
Electricity, gas and water supply & 4,835 & 5,561 & 5,705 & 5,920 & 6,737 \\
Construction & 3,709 & 4,196 & 3,877 & 4,354 & 5,161 \\
Services & 3,851 & 4,472 & 4,961 & 5,401 & 6,152 \\
Wholesale and retail trade & 3,112 & 3,627 & 4,302 & 4,662 & 5,416 \\
Hotels and restaurants & 2,340 & 2,624 & 2,336 & 2,990 & 3,612 \\
Transport, storage and communications & 4,425 & 5,122 & 5,534 & 5,991 & 6,472 \\
Real estate, renting and business activities & 4,078 & 4,566 & 5,014 & 5,003 & 6,407 \\
Financial intermediation & 7,684 & 8,914 & 9,786 & 11,002 & 12,258 \\
Public administration & 4,226 & 4,942 & 5,715 & 6,284 & 6,956 \\
Education & 2,794 & 3,370 & 3,964 & 4,200 & 4,769 \\
Health and social work & 3,089 & 3,690 & 4,154 & 4,383 & 4,781 \\
Other services & 2,913 & 3,390 & 3,840 & 4,099 & 4,697 \\
& & & & & \\
\hline
\end{tabular}

Source: National authorities; and Fund staff projections.

1/ Estimate based on quarterly data.

2/ Total average monthly wage calculated by aggregating across sectors. 
Table 8. Estonia: Employment by Sector, 1997-2001 (thousands, period average, persons aged 15 to 74)

\begin{tabular}{lrrrrr}
\hline & 1997 & 1998 & 1999 & 2000 & 2001 \\
\hline & & & & & \\
Total & 617.3 & 606.5 & 579.4 & 572.4 & 577.7 \\
& & & & & \\
Agriculture, hunting and fishing & 50.0 & 49.0 & 43.9 & 38.3 & 37.4 \\
Forestry & 6.8 & 4.8 & 3.1 & 2.9 & 2.7 \\
Mining and quarrying & 7.1 & 7.6 & 7.9 & 7.2 & 5.8 \\
Manufacturing & 136.5 & 131.8 & 122.8 & 129.2 & 134.1 \\
Electricity, gas and water supply & 16.7 & 17.2 & 16.5 & 14.7 & 11.4 \\
Construction & 44.9 & 44.1 & 38.9 & 39.7 & 39.3 \\
Services & 355.5 & 352.1 & 346.2 & 340.4 & 347.1 \\
Wholesale and retail trade & 86.1 & 85.7 & 81.9 & 79.3 & 83.6 \\
Hotels and restaurants & 14.0 & 13.7 & 13.0 & 19.9 & 17.4 \\
Transport, storage and communication & 56.6 & 55.3 & 59.4 & 56.9 & 53.7 \\
Real estate, renting and business activities & 7.1 & 8.1 & 8.6 & 7.7 & 7.2 \\
Financial intermediation & 34.0 & 37.4 & 37.4 & 40.0 & 38.2 \\
Public administration & 32.6 & 34.7 & 34.7 & 34.1 & 34.8 \\
Education & 56.7 & 54.2 & 50.3 & 44.6 & 51.0 \\
Health and social work & 35.8 & 34.0 & 31.3 & 28.5 & 30.9 \\
Other services & 32.6 & 29.0 & 29.7 & 29.6 & 30.3
\end{tabular}

Source: National authorities; and Fund staff estimates. 
Table 9. Estonia: Labor Market Indicators, 1997-2002 1/

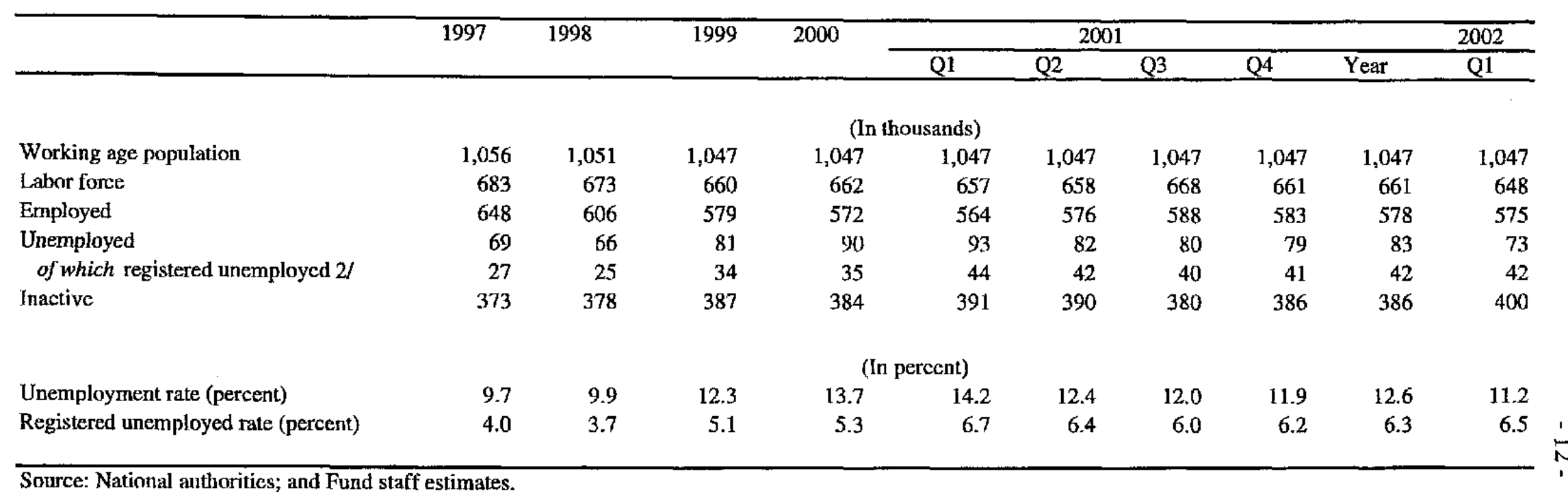

Source: National authorities; and Fund staff estimates.

1/ Labor market indicators are taken from the Estonian Labor Force Survey, except for data on registered unemployment, which are taken from the Labor Market Board.

2/ The labor force based on the Labor Force Survcy differs from the implied labor force from the Labor Market Board data. Here, the rcgistered unemployment rate from the Labor Market Board data is taken to estimate the number of registered unemployed consistent with the labor force based on the Labor Force Suryey. 
Table 10. Estonia: Unit Labor Costs by Sector, 1997-2001 1/ $(1995=100)$

\begin{tabular}{lrrrrr}
\hline & 1997 & 1998 & 1999 & 2000 & 2001 \\
\hline & & & & & \\
Total & 123.4 & 132.9 & 136.8 & 139.0 & 150.3 \\
& & & & & \\
Agriculture and hunting & 132.3 & 161.8 & 148.0 & 153.7 & 165.3 \\
Forestry & 92.4 & 92.9 & 77.6 & 72.1 & 78.4 \\
Fishing & 143.9 & 106.8 & 85.1 & 99.4 & 92.2 \\
Mining and quarrying & 94.8 & 120.8 & 149.1 & 142.3 & 125.8 \\
Manufacturing & 103.2 & 106.9 & 101.5 & 106.9 & 111.1 \\
Electricity, gas and water supply 2/ & 156.1 & 201.8 & 214.5 & 197.4 & 173.3 \\
Construction & 147.5 & 137.2 & 122.6 & 131.6 & 146.2 \\
Wholesale and retail trade & 141.3 & 155.1 & 175.1 & 170.7 & 196.3 \\
Hotels and restaurants 3/ & 97.7 & 94.4 & 71.7 & 134.7 & 130.7 \\
Transport, storage and communications & 96.2 & 99.2 & 109.2 & 105.4 & 100.6 \\
Real estate, renting and business acrivities & 140.3 & 161.9 & 187.7 & 161.1 & 183.4 \\
Financial intermediation & 129.4 & 183.0 & 185.8 & 204.0 & 199.4 \\
Public administration & 129.5 & 160.3 & 183.7 & 196.4 & 220.2 \\
Education & 145.9 & 165.7 & 181.0 & 170.4 & 219.2 \\
Health and social work & 149.7 & 163.6 & 165.2 & 154.1 & 187.4 \\
Other services & 141.9 & 148.3 & 169.5 & 171.8 & 196.1 \\
& & & & & \\
\hline
\end{tabular}

Sources: National authorities and Fund staff estimates.

1/ Unit labor costs are defined as average tnonthly wage multiplied by employment divided by real value added.

2/ The large decrease in unit labor costs in 2001 reflects a sharp decline in numbers employed.

3/ The large increase in unit labor costs in 2000 reflects the rapid expansion of total capacity in the Tallinn area that year. 
Table 11. Estonia: Summary of Generał Govemment Operations, 1997-2002

\begin{tabular}{|c|c|c|c|c|c|c|}
\hline & 1997 & 1998 & 1999 & 2000 & 2001 & 2002 \\
\hline & & & & & & Q1 (Prel.) \\
\hline & \multicolumn{6}{|c|}{ (In millions of kroans) } \\
\hline Tota] revenue and grants & 25,485 & 28,887 & 29,083 & 33,062 & 36,881 & 9,251 \\
\hline Tsx revemue & 24,076 & 27,126 & 27,383 & 30,209 & 32,890 & 8,460 \\
\hline Direst tazes & 14,473 & 17,320 & 17,518 & 18,214 & 19,775 & 5,181 \\
\hline VAT & 6,686 & 6,413 & 6,419 & $B, 153$ & 8,639 & 2,189 \\
\hline Excisex & 2,401 & 2,787 & 2,685 & 2,819 & 3,434 & 837 \\
\hline Other taxes & 511 & 602 & 761 & 1,023 & 1,042 & 256 \\
\hline Nontax revenue $y$ & 1,409 & 1,761 & 1,700 & 2,594 & 3,367 & 753 \\
\hline Grants $2 /$ & 0 & 0 & 0 & 259 & 624 & 36 \\
\hline Total expenditure $1 / 2$ & 24,339 & 29,037 & 32,542 & 33,967 & 36,548 & 8,902 \\
\hline Current expenditure & 21,860 & 25,944 & 29,318 & 31,231 & 33.389 & 8,327 \\
\hline Gonds and services & 14,682 & 17,226 & 19,443 & 20,368 & 21,650 & 5,343 \\
\hline Current tratnsfers and subsidies & 6,885 & 8,381 & 9,565 & 10,568 & 11,481 & 2,904 \\
\hline Interest payments & 293 & 339 & 309 & 295 & 258 & 79 \\
\hline Capitsl expenditure & 2,479 & 3,093 & 3,225 & 2,736 & 3,158 & 575 \\
\hline Financial strplus $(+) /$ ieficit $(-)$ & 1,146 & -150 & $-3,459$ & 905 & 333 & 349 \\
\hline Net lending $(-) 3 t$ & 264 & -77 & -81 & 329 & 63 & 33 \\
\hline Overall balarce & 1,410 & -227 & $-3,540$ & -576 & 396 & 383 \\
\hline Botrawing requirement & $-1,410$ & 227 & 3,540 & 576 & -396 & -383 \\
\hline Domestic financing, of which: & -753 & 711 & 3,888 & 919 & 1,639 & -353 \\
\hline Privatization proceeds & 155 & 25 & 3,024 & 812 & 1,970 & 387 \\
\hline Foreign financing, of which: & -657 & -483 & -349 & -343 & $-2,035$ & -30 \\
\hline \multirow[t]{2}{*}{ Change in gavermment deposits held abroad (s, increase) } & -300 & -565 & .431 & -23 & $\cdot 1,841$ & -18 \\
\hline & \multicolumn{6}{|c|}{ (In peraent of GDP) } \\
\hline Totul tevenue and grants & 39.8 & 39.3 & 38.1 & 38.7 & 38.7 & 39.9 \\
\hline Tax retentue & 37.6 & 36.9 & 35.9 & 35.4 & 34.5 & 36.5 \\
\hline Direct taxes & 22.6 & 23.6 & 23.0 & 21.3 & 20.8 & 22.3 \\
\hline VAT & 10.4 & 8.7 & 8,4 & 9.5 & 9.1 & 9.4 \\
\hline Excises & 3.7 & 3.8 & 3.5 & 3.3 & 3.6 & 3.6 \\
\hline Other taxes & 0.8 & 0.8 & 1.0 & 1.2 & 1.1 & 1.1 \\
\hline Nontax revenue 1 & 2.2 & 2.4 & 2.2 & 3.0 & 3.5 & 3.2 \\
\hline Grants $2 f$ & 0.0 & 0.0 & 0.0 & 0.3 & 0.7 & 0.2 \\
\hline Total expenditure $1 / 2 t$ & 38.0 & 39.5 & 42.6 & 39.8 & 38.4 & 38.4 \\
\hline Current expenditure & 34.1 & 35.3 & 38.4 & 36.6 & 35.0 & 35.9 \\
\hline Goods and services & 22.9 & 23.4 & 25.5 & 23.8 & 22.7 & 23.0 \\
\hline Current transfers and stubsidies & 10.8 & 11.4 & 12.5 & 12.4 & 12.1 & 12.5 \\
\hline hiterest payments & 0.5 & 0.5 & 0.4 & 0.3 & 0.3 & 0.3 \\
\hline Capital expenditure & 3.9 & 4.2 & 4.2 & 3.2 & 3.3 & 2.5 \\
\hline Finmeial surpius $(+) /$ deficit $(\cdot)$ & 1.8 & -0.2 & -4.5 & -1.1 & 0.3 & 1.5 \\
\hline Net lending $(-) 3$ & 0.4 & -0.1 & .0 .1 & 0.4 & 0.1 & 0.1 \\
\hline Overall balance & 2.2 & +0.3 & -4.6 & -0.7 & 0.4 & 1.6 \\
\hline Borrowing requirement & -2.2 & 0.3 & 4.6 & 0.7 & -0.4 & -1.6 \\
\hline Domestic fimancing, of which: & -1.2 & 1.0 & 5.1 & 1.1 & 1.7 & .1 .5 \\
\hline Privatization praceeds $3 y$ & 0.2 & D.D & 4.0 & 1.0 & 2.1 & 1.7 \\
\hline Fortign fmancing, of which: & -1.0 & -0.7 & -0.5 & -0.4 & -2.1 & -0.1 \\
\hline Change in govemment deposits held abroad (f, increake) & -1.1 & -0.8 & -0.6 & $0, \mathrm{~B}$ & .1 .9 & -0.1 \\
\hline \multicolumn{7}{|l|}{ Memarandizm items: } \\
\hline GDP (in milliom of kroons) & 64,045 & 73,538 & 76,327 & 85,496 & 95,275 & 23,205 \\
\hline
\end{tabular}


$-15$

Table 12. Estonia: Generai Government Revenue, 1997-2002

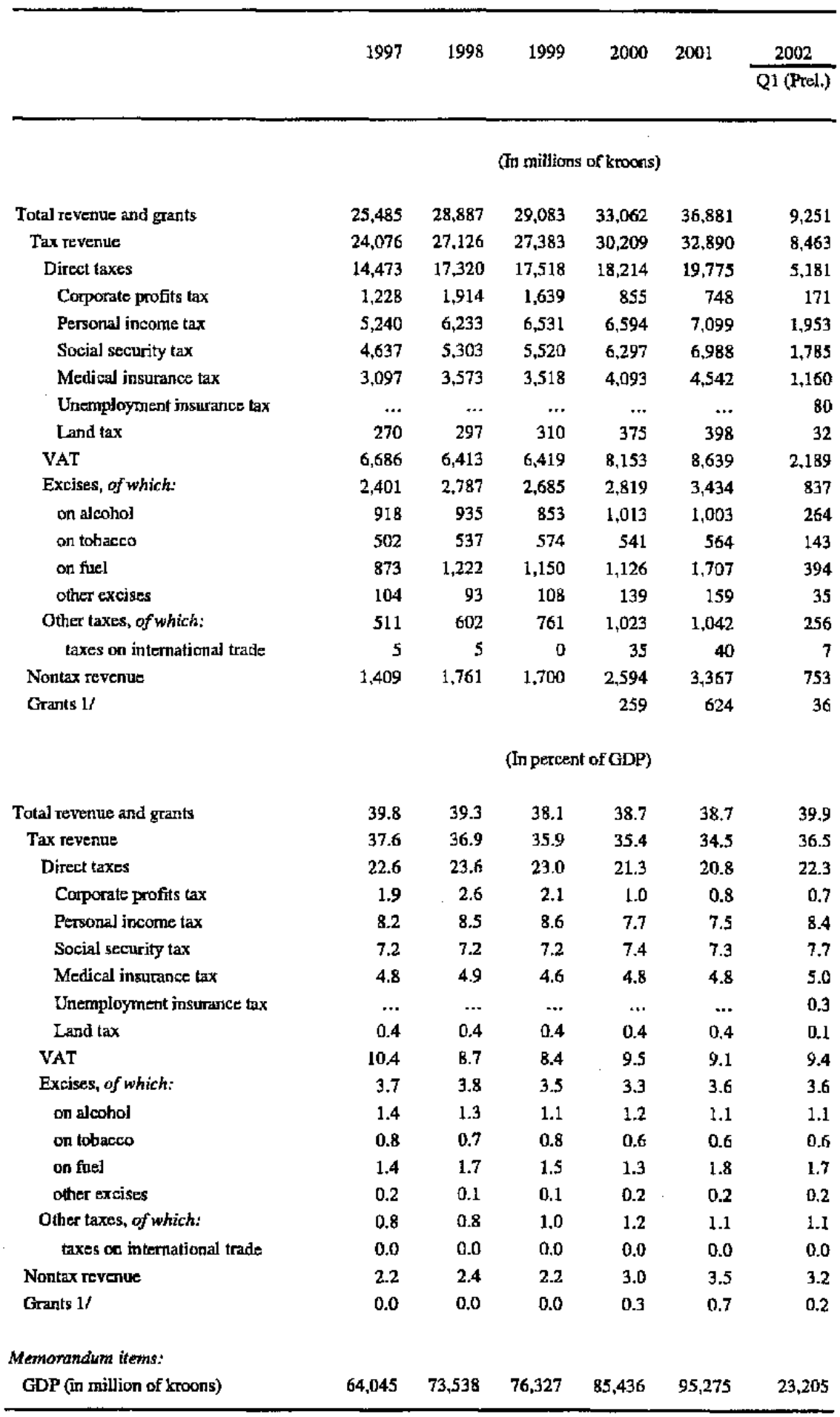

Sources: Estorian authorities; and Fund slaff estimates.

1/ Foreign grants (reflecting pre-accession financisl support from the EU) and grant-financed expenditures have been reported in the governinent finarcial stalistics since August 2001. Figures for 2000 are staff estimates. 
Table 13. Estonia: General Government Expenditure, 1997-2002

\begin{tabular}{|c|c|c|c|c|c|c|}
\hline & 1997 & 1998 & 1999 & 2000 & 2001 & 2002 \\
\hline & & & & & & Q1 (Prel.) \\
\hline & \multicolumn{6}{|c|}{ (In millions of kroons) } \\
\hline Total expenditure and net lending & 24,075 & 29,114 & 32,623 & 33,638 & 36,485 & 8,869 \\
\hline Total expenditure & 24,339 & 29,037 & 32,542 & 33,967 & 36,548 & 8,902 \\
\hline Current expenditure & 21,860 & 25,944 & 29,318 & 31,231 & 33,389 & 8,327 \\
\hline Expenditure on goods and services & 14,682 & 17,226 & 19,443 & 20,368 & 21,650 & 5,343 \\
\hline Wages and salaries $1 /$ & 5,308 & 6,775 & 8,149 & 8,718 & 9,052 & 2,199 \\
\hline Other purchases of goods and services & 9,374 & 10,450 & 11,294 & 11,650 & 12,599 & 3,145 \\
\hline Current transfers and subsidies & 6,885 & 8,381 & 9,565 & 10,568 & 11,481 & 2,904 \\
\hline Subsidies & 196 & 693 & 690 & 682 & 805 & 185 \\
\hline Transfers to households o/w & 6,689 & 7,688 & 8,876 & 9,886 & 10,676 & 2,719 \\
\hline Pensions & 4,628 & 5,200 & 6,425 & 6,445 & 6,610 & 1,607 \\
\hline Family benefits & 935 & 1,159 & 1,156 & 1,317 & 1,314 & 327 \\
\hline Sickness benefits & 552 & 662 & 607 & 716 & 738 & 216 \\
\hline Unemployment benefits & 71 & 57 & 120 & 120 & 133 & 30 \\
\hline Income mainlenance & 431 & 390 & 314 & 315 & 358 & 103 \\
\hline Other & 72 & 221 & 252 & 974 & 1,524 & 436 \\
\hline Interest payments & 293 & 339 & 309 & 295 & 258 & 79 \\
\hline Capital expenditure & 2,479 & 3,093 & 3,225 & 2,736 & 3,158 & 575 \\
\hline \multirow[t]{2}{*}{ Net lending (-) $2 /$} & 264 & -77 & -81 & 329 & 63 & 33 \\
\hline & \multicolumn{6}{|c|}{ (In percent of GDP) } \\
\hline Total expenditure and net lending & 37.6 & 39.6 & 42.7 & 39.4 & 38.3 & 38.2 \\
\hline Total expenditure & 38.0 & 39.5 & 42.6 & 39.8 & 38.4 & 38.4 \\
\hline Current expenditure & 34.1 & 35.3 & 38.4 & 36.6 & 35.0 & 35.9 \\
\hline Expenditure on goods and services & 22.9 & 23.4 & 25.5 & 23.8 & 22.7 & 23.0 \\
\hline Wages and salaries $1 /$ & 8.3 & 9.2 & 10.7 & 10.2 & 9.5 & 9.5 \\
\hline Other purchases of goods and services & 14.6 & 14.2 & 14.8 & 13.6 & 13.2 & 13.6 \\
\hline Current transfers and subsidies & 10.8 & 11.4 & 12.5 & 12.4 & 12.1 & 12.5 \\
\hline Subsidies & 0.3 & 0.9 & 0.9 & 0.8 & 0.8 & 0.8 \\
\hline Transport & 0.3 & 0.4 & 0.5 & 0.4 & 0.0 & 0.0 \\
\hline Transfers to households & 10.4 & 10.5 & 11.6 & 11.6 & 11.2 & 11.7 \\
\hline Pensions & 7.2 & 7.1 & 8.4 & 7.5 & 6.9 & 6.9 \\
\hline Family benefits & 1.5 & 1.6 & 1.5 & 1.5 & 1.4 & 1.4 \\
\hline Sickness benefits & 0.9 & 0.9 & 0.8 & 0.8 & 0.8 & 0.9 \\
\hline Unemployment benefits & 0.1 & 0.1 & 0.2 & 0.1 & 0.1 & 0.1 \\
\hline Income maintenance & 0.7 & 0.5 & 0.4 & 0.4 & 0.4 & 0.4 \\
\hline Other & 0.1 & 0.3 & 0.3 & 1.1 & 1.6 & 1.9 \\
\hline Interest payments & 0.5 & 0.5 & 0.4 & 0.3 & 0.3 & 0.3 \\
\hline Capital expenditure & 3.9 & 4.2 & 4.2 & 3.2 & 3.3 & 2.5 \\
\hline Net lending $(-) 2 /$ & 0.4 & -0.1 & -0.1 & 0.4 & 0.1 & 0.1 \\
\hline GDP (in million of kroons) & 64,045 & 73,538 & 76,327 & 85,436 & 95,275 & 23,205 \\
\hline
\end{tabular}

Sources: Estonian authorities; and Fund staff estimates.

1/ Wages and salaries of a number of budgetary institutions are included under "other goods and services". 2/ Net lending in 2000 includes EEK 303 million recovery of deposits from Maapank and EVEA bank. 
Table 14. Estonia: Fiscal Balances by Government Sector, 1997-2001

(In millions of kroons)

\begin{tabular}{|c|c|c|c|c|c|}
\hline & 1997 & 1998 & 1999 & 2000 & 2001 \\
\hline \multicolumn{6}{|l|}{ Central goverument } \\
\hline Revenue & 14,640 & 15,358 & 15,762 & $.17,683$ & 19,653 \\
\hline Tax revenue & 12,721 & 13,974 & 13,706 & 14,886 & 16,128 \\
\hline Non-tax revenue & 1,919 & 1,384 & 2,056 & 2.796 & 3.525 \\
\hline Expenditure & 13,199 & 15,415 & 17,601 & 18.415 & 19,323 \\
\hline Cutrent & 11.537 & 13,539 & 15,639 & 16,787 & 17,918 \\
\hline of which: inter-goverumental transfers & 2,467 & 2,799 & 1,875 & 2,060 & 3,172 \\
\hline Capital & 1,663 & 1,876 & 1,926 & 1,628 & 1,405 \\
\hline of which: inter-goverumental transfers & 198 & 293 & 470 & 234 & 191 \\
\hline Domestic deficit $(-/$ surplus $(+)$ & 1,441 & -57 & $-1,839$ & -733 & 330 \\
\hline Capital expendinure (foreign financed) & 103 & 128 & 234 & 14 & 63 \\
\hline Net lending (-) $1 /$ & -50 & 97 & 37 & -645 & -120 \\
\hline of which: net lending to local goveruments & $\ldots$ & $\ldots$ & 43 & -258 & -67 \\
\hline Overall deficit $(-) /$ surplus $(+)$ & 1,387 & -282 & $-2,110$ & -102 & 387 \\
\hline \multicolumn{6}{|l|}{ Local govermiment } \\
\hline Revenue and transfers & 5,382 & 6,252 & 6,597 & 6,667 & 9,064 \\
\hline Revenue (owr) & 4,052 & 4,634 & 4,752 & 4,963 & 5,701 \\
\hline Intergovernmental transfers & 1,330 & 1,618 & 1,844 & 1,704 & 3,363 \\
\hline Expenditure & 5,440 & 6,411 & 6,978 & 6,921 & 9,656 \\
\hline Cument & 4,372 & 4,968 & 5,364 & 5,593 & 7,375 \\
\hline Capital & 1,068 & 1,443 & 1,614 & 1,328 & 1,881 \\
\hline Overall Deficit $(-) /$ surphus $(+)$ & -59 & -159 & -382 & -255 & .592 \\
\hline \multicolumn{6}{|l|}{ Social Insurance Fund $2 /$} \\
\hline Revenue & 5,866 & 6.781 & 5,712 & 6,555 & 6,988 \\
\hline of which: inter-govemmental transfers & 1,506 & 1,403 & 295 & 389 & 376 \\
\hline Expenditure & 5,720 & 6,563 & 6,460 & 6,476 & 6,644 \\
\hline Defícit (-)/ surplus (+) & 147 & 217 & .749 & 80 & 344 \\
\hline \multicolumn{6}{|l|}{ Medical Insurance Fund } \\
\hline Revenue & 3,143 & 3,627 & 3,613 & 4,094 & 4,566 \\
\hline Expenditure & 2,965 & 3,618 & 3,919 & 4,084 & 4,242 \\
\hline Deficit $(-/ /$ surplus $(+)$ & 177 & 9 & -306 & 10 & 324 \\
\hline \multicolumn{6}{|l|}{ Forestry Fund 3 / } \\
\hline Revenue & 494 & 677 & $\ldots$ & $\ldots$ & $\ldots$ \\
\hline Expenditure & $4 B 0$ & 668 & ... & ... & $\ldots$ \\
\hline Deficit $(-/ /$ supplus $(+)$ & 14 & 9 & $\cdots$ & ... & $\cdots$ \\
\hline \multicolumn{6}{|l|}{ Environnental Fund 3 / } \\
\hline Reverue & 128 & 137 & ... & $\cdots$ & ... \\
\hline Expenditure & 160 & 157 & $\cdots$ & $\cdots$ & ... \\
\hline Deficit $(-) /$ surplus (t) & -32 & -21 & ... & $\cdots$ & $\cdots$ \\
\hline Overall general government deficit $(-)$ /surplus ( $(+)$ & 1,410 & -227 & $-3,540$ & -576 & 396 \\
\hline \multicolumn{6}{|l|}{ Memorandum items: } \\
\hline Nominal GDP & 64,045 & 73,538 & 76,327 & B5,436 & 95.275 \\
\hline
\end{tabular}

Source: Estonian authorities; and Fund staff estimates.

1/ Net lending in 2000 includes EEK 303 million recovery of deposits from Maapank and EVEA bank.

$2 /$ Prior to 1999 , includes payment of family benefits financed through transfers froms central govenment.

3/ The environmental and the forestry funds were closed in 1999. 
Table 15. Estonia: Fiscal Balances by Government Sector, 1997-2001

(In percent of GDP)

\begin{tabular}{|c|c|c|c|c|c|}
\hline & 1997 & 1998 & 1999 & 2000 & 2001 \\
\hline \multicolumn{6}{|l|}{ Central govemment } \\
\hline Revenue & 22.9 & 20.9 & 20.7 & 20.7 & 20.6 \\
\hline Tax reverue & 19.9 & 19.0 & 18.0 & 17.4 & 16.9 \\
\hline Nontax revenue & 3.0 & 1.9 & 2.7 & 3.3 & 3.7 \\
\hline Expenditure & 20.6 & 21.0 & 23.1 & 21.6 & 20.3 \\
\hline Current & 18.0 & 18.4 & 20.5 & 19.6 & 18.8 \\
\hline of which: inter-governmental transfers & 3.9 & 3.8 & 2.5 & 2.4 & 3.3 \\
\hline Capital & 2.6 & 2.6 & 2.5 & 1.9 & 1.5 \\
\hline of which: inter-govemmental transfers & 0.3 & 0.4 & 0.6 & 0.3 & 0.2 \\
\hline Domestic deficit $(-$ Ysurplus $(+)$ & 2.2 & -0.1 & -2.4 & -0.9 & 0.3 \\
\hline Capital expenditure (foreign financed) & 0.2 & 0.2 & 0.3 & 0.0 & 0.1 \\
\hline Net lending $1 /$ & -0.1 & 0.1 & 0.0 & -0.8 & -0.1 \\
\hline of. which: net lending to local govemuments & $\cdots$ & $\cdots$ & 0.1 & -0.3 & -0.1 \\
\hline Overall deficit (-)/ surplus (+) & 2.2 & -0.4 & -2.8 & +0.1 & 0.4 \\
\hline \multicolumn{6}{|l|}{ Local government } \\
\hline Revenue and transfers & 8.4 & 8.5 & 8.6 & 7.8 & 9.5 \\
\hline Revenue (own) & 6.3 & 6.3 & 6.2 & 5.8 & 6.0 \\
\hline Intergovernmental tcansfers & 2.1 & 2.2 & 2.4 & 2.0 & 3.5 \\
\hline Expenditure & 8.5 & 8.7 & 9.1 & 8.1 & 10.1 \\
\hline Current & 6.8 & 6.8 & 7.0 & 6.5 & 8.2 \\
\hline Capital & 1.7 & 2.0 & 2.1 & 1.6 & 2.0 \\
\hline Overall Deficit $(-y /$ surplus (+) & -0.1 & -0.2 & -0.5 & -0.3 & -0.6 \\
\hline \multicolumn{6}{|l|}{ Social Insurance Fund $2 /$} \\
\hline Revenue $3 /$ & 92 & 9.2 & 7.5 & 7.7 & 7.3 \\
\hline of which: inter-governmental transfers & 2.4 & 1.9 & 0.4 & 0.5 & 0.4 \\
\hline Expenditure & 8.9 & 8.9 & 8.5 & 7.6 & 7.0 \\
\hline Deficit & 0.2 & 0.3 & -1.0 & 0.1 & 0.4 \\
\hline \multicolumn{6}{|l|}{ Medical Insurance Fund } \\
\hline Revenue $3 /$ & 4.9 & 4.9 & 4.7 & 4.8 & 4.8 \\
\hline Expenditure & 4.6 & 4.9 & 5.1 & 4.8 & 4.5 \\
\hline Deficit & 0.3 & 0.0 & -0.4 & 0.0 & 0.3 \\
\hline \multicolumn{6}{|l|}{ Forestry Fund 4/ } \\
\hline Revente & 0.8 & 0.9 & $\cdots$ & $\cdots$ & $\cdots$ \\
\hline Expenditure & 0.7 & 0.9 & $\cdots$ & $\cdots$ & $\ldots$ \\
\hline Deficit & 0.0 & 0.0 & $\cdots$ & $\cdots$ & $\cdots$ \\
\hline \multicolumn{6}{|l|}{ Environmental Fund $4 /$} \\
\hline Revenue & 0.2 & 0.2 & $\ldots$ & $\ldots$ & $\cdots$ \\
\hline Expenditure & 0.2 & 0.2 & $\cdots$ & $\cdots$ & $\cdots$ \\
\hline Deficit & 0.0 & 0.0 & $\cdots$ & $\cdots$ & $\cdots$ \\
\hline General government deficit/surplus & 2.2 & -0.3 & -4.6 & -0.7 & 0.4 \\
\hline \multicolumn{6}{|l|}{ Memorandum item: } \\
\hline Nominai GDP (in milliuns of kroons) & 64,045 & 73,538 & 76,327 & 85,436 & 95,275 \\
\hline
\end{tabular}

Source: Estonian authorities; and Fund staff estimates.

1/ Net lending in 2000 includes EEK 303 million recovery of deposits from Maapank and EVEA bank.

2/ Prior to 1999, includes payment of farnily benefits financed through transfers from central government.

3/ The environmental and the forestry funds were closed in 1999. 
$-19-$

Table L6. Istonia Banking Survey and Monetary Anchorities: 1997-2002 l/ (in milltons of EEK, unless otherwise indicated)

\begin{tabular}{|c|c|c|c|c|c|c|}
\hline & 1997 & 1998 & 1999 & 2000 & 2001 & $\frac{2002}{\mathrm{Mrx}}$ \\
\hline \multicolumn{7}{|l|}{ Banking Survey } \\
\hline Nel foreiga assets & 5.083 & 5.112 & 8,022 & 9,098 & 12.285 & $116 \leq 5$ \\
\hline Nel forrign assets (BOE) & 10,096 & 10,493 & 12,952 & 15,167 & 14,319 & 13644 \\
\hline Pureign assets $2 / 3$ & 10,901 & 10,909 & 13,334 & 15,540 & 14,573 & 15476 \\
\hline Toreign iiabilities & -805 & -415 & -402 & 373 & -254 & -1832 \\
\hline Net fareign assets (comrnercial banks) & -.5 .913 & $-5,381$ & $-4,910$ & $-6,068$ & -2.034 & -1988 \\
\hline Forrign assets & 8,078 & 6,481 & 8,772 & 10,359 & 15.475 & 17040 \\
\hline Foreiga Liabillter ex & $-13,090$ & $-11,862$ & $-13,682$ & $-16,427$ & $-17,510$ & -19028 \\
\hline Net dorneslic assets & 15.383 & 16.216 & 18,368 & 24,064 & 28,518 & 29267 \\
\hline Damestie eredit & 20,797 & 24,223 & 26,542 & 33,758 & 41.994 & 43169 \\
\hline Net eredit to general government & -1.716 & .930 & -197 & $-1,078$ & -535 & -1475 \\
\hline Credit to governmest (banks) & 1.108 & 955 & 1,172 & 1.268 & 1.742 & 1696 \\
\hline Goverrment deposils (banks) & $-2,473$ & $-1,882$ & $-1,345$ & -2.343 & 2,311 & -3166 \\
\hline Net Gredit to government (BOE) & 4 & 3 & 3 & 3 & 1 & 1 \\
\hline Oaverfment deposits (BOE) & -355 & -6 & -27 & .7 & -8 & -7 \\
\hline Credit to thongoverament & 22,512 & 25.153 & 26.739 & 34.837 & 42,570 & 44544 \\
\hline Credit to nonfinancial public enterprises & 329 & 226 & 372 & 263 & 142 & 112 \\
\hline Credit so private sector & 16,953 & 18.590 & 19,877 & 22,200 & 26,321 & 27245 \\
\hline Credit to enterprises & 12,812 & 14,369 & 14,571 & 15,376 & 17,161 & 17619 \\
\hline Credil to households & 4,141 & 4,221 & 5.306 & 6,827 & 9,160 & 9625 \\
\hline Credit to nonbenk financial institutions & 5.231 & 6,337 & $6.48 y$ & 12.370 & 16.107 & 17288 \\
\hline Other itsms (net) & -5.414 & $-8,007$ & -8.174 & $.9,695$ & $-13,476$ & $-1390 \mid$ \\
\hline Brosd money & 20,466 & 21,328 & 26,390 & 33.162 & 40.803 & 40929 \\
\hline $\mathrm{Mi}$ & 13,998 & 13.120 & 17.336 & 20.869 & 24.948 & 23964 \\
\hline Cumency outside bariks & 4,588 & 4,539 & 5.711 & 6.201 & 6,952 & 6848 \\
\hline Demand defnsits & 9,410 & 8581 & 11,624 & 14,668 & 17,996 & 17116 \\
\hline Tirste and savipgst deposits & 6.468 & 8.208 & 9,054 & 12.293 & 15.855 & 16958 \\
\hline \multicolumn{7}{|l|}{ Monetary Authonities } \\
\hline Net forrign assels & 10,096 & 10,493 & 12,932 & 15,167 & 14.319 & $\mathrm{t} 36 \pm 4$ \\
\hline Foreign assets $2 / 3 /$ & 10,901 & 10,909 & 13,334 & 15,540 & 14.573 & 15476 \\
\hline of which: currency board cover 4 l & B.527 & 9,070 & 11,526 & 13,207 & 11.910 & 11123 \\
\hline Foreign iabilities & -805 & 415 & -402 & -373 & -254 & -1832 \\
\hline Net domescic assets & -1.569 & $-6,423$ & $-i, 406$ & -1.960 & $-2,409$ & -2520 \\
\hline Net claims on Goverament & $-35 !$ & -3 & -24 & -4 & -6 & -5 \\
\hline Cyaims on tinanciul institutions & 196 & $28 \mathrm{t}$ & 268 & 10 & 8 & 8 \\
\hline Gaims on private sector & 44 & 57 & 66 & 30 & 75 & 75 \\
\hline Other & $-1,448$ & $-1,758$ & $\cdot 1.716$ & $-2,035$ & -2486 & -2599 \\
\hline Base money & 8,523 & 9,070 & 11,526 & 13,207 & $\lfloor, 910$ & 11123 \\
\hline Currenty istue & 5,439 & 5.291 & 6.649 & 7.277 & 8,067 & 7735 \\
\hline Deposits of commerciel banks & 3.035 & 3,676 & 4,824 & 5,718 & 3.644 & 9355 \\
\hline of which: required reserves $5 /$ & 2.000 & 2,675 & 2.734 & 4,634 & 5.904 & 6140 \\
\hline OUter deposils at BDE & s3 & 4 & 54 & 211 & 28 & 31 \\
\hline \multicolumn{7}{|l|}{ Memonortum irens: } \\
\hline Base mnney mulbiplier & 2.40 & 2.35 & 2.29 & $25:$ & 3.43 & 3.68 \\
\hline Currency-tn-depposit mutú & 0.29 & 0.27 & 0.28 & 0.23 & 0.21 & 0.20 \\
\hline Bank reservas-to-depasit ratio & 0.25 & 0.27 & 0.28 & 0.25 & 0.14 & 0.12 \\
\hline Velodty (period average) & 3.54 & 3.39 & 3.14 & 2.78 & 2.57 & $\ldots$ \\
\hline Gtoss international neserves (in milliusts of US\$) 2 & 759 & 813 & 851 & 992 & 823 & 861 \\
\hline Net internatiatral meserves (in millions of turo) $6 J$ & 105 & 124 & 115 & 149 & 179 & 179 \\
\hline \multirow[t]{2}{*}{ Governmenl balancas hedó abroul $7 /$} & 700 & 1.224 & 1,995 & 1,447 & $\cdots$ & $\cdots$ \\
\hline & \multicolumn{6}{|c|}{ (persentage change from same period in preceding year) } \\
\hline Net fortign assere of banking aystent & -24.4 & 0.6 & 56.9 & 13.4 & 95.0 & :7.3 \\
\hline Net dcamleylic asets of banking system & $8 \% .2$ & 5.4 & 13.3 & 31.0 & 18,5 & 16.3 \\
\hline Cyedit of banking system va non-govenment & 79.0 & 11.7 & 6.3 & 30.3 & 22.2 & 23.1 \\
\hline Credit to the private sector & 67.3 & 9.7 & 6.9 & 11.7 & 18.5 & 20.6 \\
\hline Credit to nanbank financingl institutions & 144.5 & 21.1 & 2.4 & 90.6 & 30.2 & 28.5 \\
\hline Broad money & 37.8 & 4.2 & 23.7 & 25.7 & 23.0 & 16.6 \\
\hline MI & 24.0 & .6 .3 & 32.1 & 20.4 & 19.5 & 16.2 \\
\hline Base raontey & 37.7 & 6.4 & 27.1 & 14.6 & -9.8 & 5.6 \\
\hline
\end{tabular}

Source: Bank of Estonia and Fund siaff estimates

1/The monetary anthxities' accoumts and the manelary survey have been revised, following the recommendations of the (1999 STA nission on money and barking sistigtics. The mein changes affect the menthly revahation of the monetary authorities' gold, the coverage of government entities and depositary institutions, and the inclusiou of finsncisl derivatives in the balance sheel of commercial banks

2/ Excludes foreign sesets of the central gavermment's Siahilizsiñon Reserve Funte

3/ The Bank of Estomia's furtign assels ross shapply in December I 999 as comunercid benks shiftod fonds into their accounts with the Bank of Eatonis to thance domertic liquidity in anticipation of Y2K problems.

4/ Currency board cover is equivalent to base money (e.g., the sum of curreney issue plus the koon jiabilities of tho Bank af Estania in its

eorrespondent aciounts).

5/ Requirement to be met on the basis of daily average of deposits aves Inoneh. Up to June 2000, it includes liquidity requirement equivalent to 3

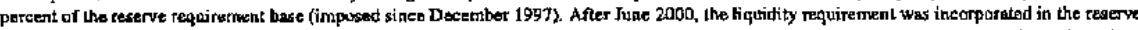
requirement. Starting in January 2001, 3 pereentsge points of the i 3 percent reserve requirement can be met trith high quality curo-denominated foreign instrunents.

6f Net of correney brand exver (prograsn thefinition).

$7 /$ Including balances in Bie Stabilization Reserve Fund (SRF). 
Table 17. Estonia: Maturity and Currency Composition of Deposits, 1997-2002

\begin{tabular}{|c|c|c|c|c|c|c|}
\hline & 1997 & 1998 & 1999 & 2000 & 2001 & 2002 \\
\hline & & & & & & Mar \\
\hline & \multicolumn{6}{|c|}{ (In millions of kroons) } \\
\hline Total deposits & 21,401 & 21,470 & 26,412 & 34,773 & 42,680 & 43,529 \\
\hline \multicolumn{7}{|l|}{ Of which: } \\
\hline Demand Deposits & 14,500 & 12,763 & 16,627 & 21,198 & 26,762 & 25,863 \\
\hline In EEK & 10,744 & 9,826 & 12,490 & 15,722 & 19,585 & 18,585 \\
\hline In Foreign Currency & 3,756 & 2,938 & 4,137 & 5,476 & 7,177 & 7,277 \\
\hline Tine Deposits & 6,901 & 8,707 & 9,784 & 13,575 & 15,918 & 17,667 \\
\hline In EEK & 4,871 & 5,572 & 5,713 & 7,243 & 10,244 & 12,093 \\
\hline \multicolumn{7}{|l|}{ Of which: } \\
\hline up to 3 months & 1,799 & 1,985 & 1,830 & 2,257 & 3,074 & 4,420 \\
\hline 3-6 months & 476 & 615 & 718 & 913 & 1,493 & 1,637 \\
\hline $6-12$ months & 1,805 & 2,373 & 2,463 & 3,085 & 4,526 & 4,882 \\
\hline over 1 year & 789 & 599 & 694 & 973 & 1,121 & 1,111 \\
\hline In Foreign Currency & 2,029 & 3,135 & 4,071 & 6,332 & 5,675 & 5,573 \\
\hline \multicolumn{7}{|l|}{ Of which: } \\
\hline up to 3 months & 965 & 1,358 & 1,667 & 2,932 & 2,620 & 2,524 \\
\hline 3-6 months & 396 & 347 & 417 & 668 & 524 & 554 \\
\hline $6-12$ months & 505 & 695 & 920 & 1,432 & 1,507 & 1,467 \\
\hline \multirow[t]{2}{*}{ over I year } & 163 & 734 & 1,067 & 1,300 & 1,019 & 1,028 \\
\hline & \multicolumn{6}{|c|}{ (In percent of total deposits) } \\
\hline Demand Deposits & 67.8 & 59.4 & 63.0 & 61.0 & 62.7 & 59.4 \\
\hline In EEK & 50.2 & 45.8 & 47.3 & 45.2 & 45.9 & 42.7 \\
\hline In Foreign Currency & 17.5 & 13.7 & 15.7 & 15.7 & 16.8 & 16.7 \\
\hline Time Deposits & 32.2 & 40.6 & 37.0 & 39.0 & 37.3 & 40.6 \\
\hline In EEK & 22.8 & 26.0 & 21.6 & 20.8 & 24.0 & 27.8 \\
\hline \multicolumn{7}{|l|}{ of which: } \\
\hline up to 3 months & 8.4 & 9.2 & 6.9 & 6.5 & 7.2 & 10.2 \\
\hline 3-6 months & 2.2 & 2.9 & 2.7 & 2.6 & 3.5 & 3.8 \\
\hline 6-12 months & 8.4 & 11.1 & 9.3 & 8.9 & 10.6 & 11.2 \\
\hline over 1 year & 3.7 & 2.8 & 2.6 & 2.8 & 2.6 & 2.6 \\
\hline In Foreign Currency & 9.5 & 14.6 & 15.4 & 18.2 & 13.3 & 12.8 \\
\hline \multicolumn{7}{|l|}{ Of which: } \\
\hline up to 3 months & 4.5 & 6.3 & 6.3 & 8.4 & 6.1 & 5.8 \\
\hline 3-6 months & 1.8 & 1.6 & 1.6 & 1.9 & 1.2 & 1.3 \\
\hline 6-12 months & 2.4 & 3.2 & 3.5 & 4.1 & 3.5 & 3.4 \\
\hline over 1 year & 0.8 & 3.4 & 4.0 & 3.7 & 2.4 & 2.4 \\
\hline \multicolumn{7}{|l|}{ Memorandum Items: } \\
\hline Total foreign currency deposits (in EEK millions) & 5,785 & 6,073 & 8,208 & 11,808 & 12,852 & 12,851 \\
\hline Of which: & \multicolumn{6}{|c|}{ (In percent of total foreign currency deposits) } \\
\hline DM/euro $1 /$ & 27.1 & 44.4 & 35.7 & 31.8 & 31.9 & 30.5 \\
\hline US \$ & 64.2 & 47.1 & 58.7 & 63.0 & 62.4 & 64.8 \\
\hline Other & 8.6 & 8.5 & 5.6 & 5.2 & 5.6 & 4.7 \\
\hline
\end{tabular}

Source: Bank of Estonia.

1/ After January 1, 1999 includes deposits denominated in euros. 
Table 18. Estonia: Maturity and Currency Composition of Loans, 1997-2002

\begin{tabular}{|c|c|c|c|c|c|c|}
\hline & 1997 & 1998 & 1999 & 2000 & 2001 & 2002 \\
\hline & & & & & & Mar \\
\hline & & & In million & of kroons) & & \\
\hline Total loans & 21,295 & 23,898 & 26,660 & 34,237 & 40,693 & 42,629 \\
\hline Of which: & & & & & & \\
\hline Short term loans (one year and less) $1 /$ & 4,888 & 3,201 & 3,828 & 4,752 & 5,531 & 5,379 \\
\hline In EEK & 2,626 & 1,182 & 1,050 & 1,539 & 1,766 & 1,787 \\
\hline In Foreign Currency & 2,262 & 2,019 & 2,779 & 3,213 & 3,765 & 3,592 \\
\hline Long term loans (over one year) & 16,254 & 20,697 & 22,829 & 29,482 & 35,159 & \\
\hline In EEK & 6,210 & 4,523 & 5,309 & 6,014 & 6,893 & 6,675 \\
\hline Of which: & & & & & & \\
\hline $1-3$ years & 2,369 & 1,572 & 1,357 & 1,796 & 1,958 & 1,884 \\
\hline over 3 years & 3,841 & 2,951 & 3,952 & 4,218 & 4,935 & 4,791 \\
\hline In Foreign Currency & 10,044 & 16,174 & 17,520 & 23,468 & 28,266 & 30,572 \\
\hline Of which: & & & & & & \\
\hline 1-3 years & 3,017 & 4,743 & 4,118 & 4,949 & 4,770 & 3,725 \\
\hline over 3 years & 7,028 & 11,432 & 13,402 & 18,519 & 23,496 & 26,847 \\
\hline & & & n percent & total loan & & \\
\hline Short term loans (one year and less) $1 /$ & 23.0 & 13.4 & 14.4 & 13.9 & 13.6 & 12.6 \\
\hline In EEK & 12.3 & 4.9 & 3.9 & 4.5 & 4.3 & 4.2 \\
\hline In Foreign Currency & 10.6 & 8.4 & 10.4 & 9.4 & 9.3 & 8.4 \\
\hline Long tem loans (over one ycar) & 76.3 & 86.6 & 85.6 & 86.1 & 86.4 & 0.0 \\
\hline In EEK & 29.2 & 18.9 & 19.9 & 17.6 & 16.9 & 15.7 \\
\hline Of which: & & & & & & \\
\hline 1-3 years & 11.1 & 6.6 & 5.1 & 5.2 & 4.8 & 4.4 \\
\hline over 3 years & 18.0 & 12.3 & 14.8 & 12.3 & 12.1 & 11.2 \\
\hline In Foreign Currency & 47.2 & 67.7 & 65.7 & 68.5 & 69.5 & 71.7 \\
\hline Of which: & & & & & & \\
\hline $1-3$ years & 14.2 & 19.8 & 15.4 & 14.5 & 11.7 & 8.7 \\
\hline over 3 years & 33.0 & 47.8 & 50.3 & 54.1 & 57.7 & 63.0 \\
\hline Memorandum Items: & & & & & & \\
\hline Total foreign currency loans (in EEK millions) & 12,376 & 18,193 & 20,301 & 26,684 & 32,031 & 34,167 \\
\hline Of which: & & (In percer & t of total $\mathrm{f}$ & reign curre & ncy loans) & \\
\hline DM/euro $2 /$ & 85.1 & 89.9 & 88.0 & 86.7 & 90.0 & 93.2 \\
\hline US \$ & 11.3 & 8.2 & 10.8 & 12.5 & 9.2 & 6.7 \\
\hline Other & 3.6 & 1.9 & 1.3 & 0.8 & 0.7 & 0.1 \\
\hline & & (In perc & nt, unless & therwise i & Idicated) & \\
\hline Total assets of banking system (in EEK millions) & 40,583 & 40,995 & 47,071 & 57,822 & 68,411 & 71,262 \\
\hline Total deposits/total assets & 52.7 & 52.4 & 56.1 & 60.1 & 62.4 & 61.1 \\
\hline Total loans/total deposits & 99.5 & 111.3 & 100.9 & 98.5 & 95.3 & 97.9 \\
\hline Short-term loans/total loans & 23.0 & 13.4 & 14.4 & 13.9 & 13.0 & 12.4 \\
\hline Short-term deposits/total deposits & 67.8 & 59.4 & 63.0 & 61.0 & 62.7 & 59.4 \\
\hline
\end{tabular}

Source: Bank of Estonia.

1/ The bulk of short term loans have maturities in the 6-12 month range.

2/ After January 1, 1999 includes all loans denominated in euros. 
Table 19. Estonia: Average Interest Rates of Deposits and Loans, 1997-2002

(In percent)

\begin{tabular}{|c|c|c|c|c|c|c|c|c|c|}
\hline & \multirow[t]{2}{*}{1997} & \multirow[t]{2}{*}{1998} & \multirow[t]{2}{*}{1999} & \multirow[t]{2}{*}{2000} & \multicolumn{4}{|c|}{2001} & \multirow{2}{*}{$\frac{2002}{\mathrm{Mar}}$} \\
\hline & & & & & Mar & Jun & Sep & Dec & \\
\hline \multicolumn{10}{|l|}{ Kroon denominated } \\
\hline \multicolumn{10}{|l|}{ Deposit rates 1/ } \\
\hline Demand deposits & $\ldots$ & $\cdots$ & $\cdots$ & $\cdots$ & $\cdots$ & $\cdots$ & $\ldots$ & $\ldots$ & $\cdots$ \\
\hline \multicolumn{10}{|l|}{ Time deposits } \\
\hline up to 3 months & 12.2 & 7.9 & 2.9 & 4.4 & 4.3 & $\begin{array}{l}4.1 \\
4.8\end{array}$ & & 2.6 & $\begin{array}{l}2.6 \\
3.5\end{array}$ \\
\hline 3- 6 months & 11.1 & 10.7 & $\begin{array}{l}4.4 \\
6.1\end{array}$ & $\begin{array}{l}4.8 \\
5.3\end{array}$ & $\begin{array}{l}4.5 \\
5.5\end{array}$ & $\begin{array}{l}4.8 \\
5.1\end{array}$ & $\begin{array}{l}4.4 \\
5.0\end{array}$ & $\begin{array}{l}3.6 \\
4.0\end{array}$ & $\begin{array}{l}3.5 \\
4.0\end{array}$ \\
\hline $\begin{array}{l}6-12 \text { months } \\
\text { over } 1 \text { year }\end{array}$ & 10.8 & 8.9 & 8.9 & 6.8 & 6.0 & 5.4 & 5.5 & 4.5 & 4.3 \\
\hline \multicolumn{10}{|l|}{ Lending rates $2 /$} \\
\hline Loans up to 3 months & 19.8 & 15.9 & 8.2 & 7.5 & 7.8 & 5.9 & 6.3 & 9.4 & 8.0 \\
\hline Loans 3 to 6 months & 14.0 & 17.6 & 9.6 & 6.9 & 6.7 & 8.6 & 10,2 & 9.1 & 9.2 \\
\hline Loans 6 to 12 months & 15.0 & 17.1 & 10.2 & 8.4 & 9.8 & 9.7 & 9.9 & 9.7 & 7.9 \\
\hline Loans $1-5$ years & 12.0 & I6.5 & 7.9 & 8.2 & 11.0 & 12.1 & 11.1 & 10.5 & 11.9 \\
\hline Loans 5 to 10 years & 10.9 & 14.7 & 9.0 & 10.5 & 10.9 & 10.9 & 9.9 & 9.4 & 10.2 \\
\hline Loans over 10 years & 7.4 & 14.5 & 10.6 & 9.7 & 9.9 & 9.4 & 10.0 & 9.7 & 9.2 \\
\hline \multicolumn{10}{|l|}{ Euro denominated } \\
\hline Deposit rates $1 /$ & $\ldots$ & $\ldots$ & 3.0 & 4.5 & 4.2 & 4.0 & 3.5 & 2.7 & 2.7 \\
\hline up to 3 months & $\ldots$ & $\ldots$ & 3.0 & 4.5 & 4.2 & 4.0 & 3.6 & 2.7 & 2.7 \\
\hline 3-6 months & $\ldots$ & $\cdots$ & 3.5 & 4.6 & 4.3 & 4.1 & 3.2 & 3.0 & 3.0 \\
\hline $6-12$ months & $\ldots$ & $\ldots$ & 3.5 & 5.2 & 5.3 & 4.0 & 3.5 & 3.0 & 3.2 \\
\hline over 1 year & $\ldots$ & $\ldots$ & 3.8 & 5.3 & 5.2 & 4.5 & 3.5 & 3.4 & 3.4 \\
\hline Lending rates $2 /$ & $\ldots$ & $\ldots$ & 7.1 & 7.0 & 7.7 & 6.2 & 5.5 & 5.6 & 5.3 \\
\hline Short term & $\ldots$ & $\cdots$ & 4.6 & 6.3 & 5.6 & 5.1 & 4.4 & 4.2 & 4.2 \\
\hline Long term & ... & $\ldots$ & 7.9 & 7.4 & 8.7 & 7.2 & 6.3 & 6.5 & 6.0 \\
\hline \multicolumn{10}{|l|}{ Money market rates } \\
\hline Interbank overnight loans 3 / & 17.1 & 16.0 & $\cdots$ & 5.5 & $\ldots$ & $\ldots$ & $\ldots$ & 3.5 & $\cdots$ \\
\hline
\end{tabular}

Source: Bank of Estonia.

1/ Weighted average annual interest rates on deposits placed with commercial banks by individuals and tompanies.

2/ Weighted average annual interest rates on loans granted to individuals and companies by commercial banks.

3/ Absence of rate indicates there was no activity during that month. 
Table 20. Estonia: Nonperforming Loans of Commercial Banks, 1997-2002

\begin{tabular}{|c|c|c|c|c|c|c|}
\hline & 1997 & 1998 & 1999 & 2000 & 2001 & 2002 \\
\hline & & & & & & Mar \\
\hline & & (In $\mathrm{m}$ & ons of 1 & ons; enc & of period) & \\
\hline Total & 248 & 326 & 461 & 358 & 543 & 697 \\
\hline Overdue up to 30 days & 74 & 75 & 14 & 40 & 64 & 300 \\
\hline Overdue from $30-60$ days & 14 & 27 & 12 & 3 & 13 & 19 \\
\hline Overdue over 60 days & 160 & 224 & 435 & 315 & 466 & 379 \\
\hline & & & cent of & tal loan & ortfolio) & \\
\hline Total & 1.2 & 1.4 & 1.7 & 1.0 & 1.3 & 1.6 \\
\hline Overdue up to 30 days & 0.3 & 0.3 & 0.1 & 0.1 & 0.2 & 0.7 \\
\hline Overdue from $30-60$ days & 0.1 & 0.1 & 0.0 & 0.0 & 0.0 & 0.0 \\
\hline Overdue over 60 days & 0.8 & 0.9 & 1.6 & 0.9 & 1.1 & 0.9 \\
\hline
\end{tabular}

Source: Bank of Estonia. 
Table 21. Estonia: Commercial Bank Profits (on a consolidated basis), 1997-2002

\begin{tabular}{|c|c|c|c|c|c|c|}
\hline & 1997 & 1998 & 1999 & 2000 & 2001 & 2002 \\
\hline & \multicolumn{4}{|c|}{ Year } & & $\mathrm{Q} 1$ \\
\hline Total profits (pre-tax) & 1,069 & -501 & 657 & 625 & 1,706 & 262 \\
\hline \multicolumn{7}{|l|}{ Of which: } \\
\hline Net interest Income & 1,441 & 1,391 & 1,721 & 1,932 & 2,183 & 555 \\
\hline Interest income & 2,659 & 3,085 & 3,512 & 3,744 & 4,308 & 1,000 \\
\hline Interest expenses & 1,217 & 1,694 & 1,791 & 1,812 & 2,126 & 445 \\
\hline Net commission income & 548 & 466 & 575 & 709 & 780 & 208 \\
\hline Commission income & 799 & 693 & 826 & 965 & 1,063 & 271 \\
\hline Commission expenses & 251 & 228 & 251 & 256 & 283 & 63 \\
\hline Income from financial investments & 117 & 21 & 134 & 37 & 710 & 11 \\
\hline Net income from financial operations & 715 & -235 & 417 & 506 & 443 & 79 \\
\hline Administrative expenses & 1,244 & 1,069 & 1,319 & 1,375 & 1,584 & 413 \\
\hline Valuation adjustments & -500 & -961 & -860 & $-1,106$ & -697 & -147 \\
\hline \multicolumn{7}{|l|}{ Memorandum items: } \\
\hline Return on assets $1 /$ & 2.6 & -1.2 & 1.4 & 1.1 & 2.5 & $\ldots$ \\
\hline Return on capital $2 /$ & 19.9 & -6.4 & 7.8 & 8.6 & 18.8 & $\cdots$ \\
\hline
\end{tabular}

Sources: Bank of Estonia; and Fund staff estimates.

1/ Defined as ratio of pre-tax profits to total assets.

2/ Defined as ratio of pre-tax profits to capital. 
Table 22. Estonia: Balance of Payments 1997-2001

\begin{tabular}{|c|c|c|c|c|c|}
\hline & 1997 & 1998 & 1999 & 2000 & 2001 \\
\hline & \multicolumn{5}{|c|}{ (In millions of euro) } \\
\hline Current Account & -497 & .428 & -231 & -348 & -394 \\
\hline Trade Bafance & -996 & -997 & .773 & -862 & -882 \\
\hline Exports & 2,027 & 2,399 & 2,364 & 3,579 & 3,747 \\
\hline Imports & $-3,024$ & $-3,396$ & $-3,137$ & $-4,441$ & $-4,629$ \\
\hline Services Balence & 524 & 511 & 533 & 587 & 629 \\
\hline $\begin{array}{l}\text { Receipts } \\
\text { of which: }\end{array}$ & 1,169 & 1,321 & 1,403 & 1,628 & 1,840 \\
\hline Transportation & 581 & 636 & 658 & 785 & 886 \\
\hline Travel & 419 & 484 & 518 & 549 & 568 \\
\hline Construction & 52 & 52 & 29 & 43 & 87 \\
\hline $\begin{array}{l}\text { Payments } \\
\text { of which: }\end{array}$ & -645 & -810 & -870 & $-1,041$ & $-1,241$ \\
\hline Transportation & -593 & -719 & -694 & -891 & .545 \\
\hline Travel & -210 & -271 & .395 & .432 & -214 \\
\hline Construetion & -36 & .54 & -36 & -37 & .35 \\
\hline Income & -128 & -74 & -96 & -223 & -327 \\
\hline Employee cornpensation & 125 & 74 & 93 & 214 & 325 \\
\hline \multicolumn{6}{|l|}{ of which: } \\
\hline Direct Investment & -201 & -130 & -176 & -410 & -646 \\
\hline Portfolio Investment & -15 & 61 & 46 & 40 & 57 \\
\hline Current Transfers & 103 & 132 & 106 & 150 & 187 \\
\hline Capital and Financial Account & 697 & 436 & 378 & 399 & 366 \\
\hline Capital Transfers & 0 & 2 & 1 & 18 & 6 \\
\hline Financial Account & 697 & 435 & 377 & 381 & 360 \\
\hline Direct Invostment & 113 & 508 & 205 & 358 & 391 \\
\hline $\begin{array}{l}\text { From abroad 1/ } \\
\text { of which: }\end{array}$ & & 598 \\
\hline Equity & 170 & 708 & 319 & 491 & 376 \\
\hline Reinvested dividends & 163 & 49 & 90 & 227 & 473 \\
\hline Other capital & 129 & 253 & 147 & $\$ 13$ & 320 \\
\hline Outward (by Estonians) & -122 & -5 & .79 & -67 & -207 \\
\hline Net equity investment l/ & 33 & 57 & 222 & -31 & 49 \\
\hline \multicolumn{6}{|l|}{ of which: } \\
\hline Banks & 406 & 19 & 6 & 134 & -118 \\
\hline Government & -40 & -52 & .31 & -22 & -7 \\
\hline Monetary Authorities & -19 & -19 & -13 & -8 & -12 \\
\hline Errory and Omissions & -24 & 0 & -33 & 13 & 6 \\
\hline Overall balance & 176 & 8 & 115 & 64 & -22 \\
\hline Change in official reserves (- increase) & -176 & -8 & -115 & -64 & 22 \\
\hline Memorandum Items: & \multicolumn{5}{|c|}{ (units as indicaled) } \\
\hline Euro/LSS exchange rate (period average) & 1.13 & 1.12 & 1.12 & 1.12 & 1.11 \\
\hline \multicolumn{6}{|l|}{ Gross International Reserves $3 / 4 / 5 /$} \\
\hline (Euro millions) & 694 & 696 & 852 & 993 & 931 \\
\hline \multirow[t]{2}{*}{ In months of imports 6} & 3.2 & 3.0 & 4.1 & 3.9 & 3.2 \\
\hline & \multicolumn{5}{|c|}{ (in percent) } \\
\hline Trade Balance/GDP & -24.4 & -21.4 & -15.8 & -15.8 & -14.5 \\
\hline Current Account/GDP & .12 .2 & -9.2 & -4.7 & -6.4 & -6.5 \\
\hline
\end{tabular}

Sources: Bark of Estonia and Fund staff estingates.

I/ The large flows in 1998 were associated with the purchase by Swedish banks of substantial interests

in the two largest Estonian banks.

2f Includes operations in debt securities.

3/ Excludes Government deposits held abroad (including in the SRF).

4/ Changes in gross intersational reserves may differ from flows inplied by overall balance of payments due to valuation changes.

5/ Gross international reseryes at end-1999 were inflated by barks shifting resources from accounts abroad to the Bank of Estonia to enhance liquidity in anticipation of Y2K-related problems.

6/ Excludes inports of goods for processing. 
Table 23. Estonia: Direction of Trade - Exports by Countries 1997-2001

\begin{tabular}{|c|c|c|c|c|c|}
\hline & 1997 & 1998 & 1999 & 2000 & 2001 \\
\hline & \multicolumn{5}{|c|}{ (In millions of kroons) } \\
\hline Finland & 5,994 & 8,310 & 8,136 & 17,393 & 19,411 \\
\hline Sweden & 5,395 & 7,333 & 7,875 & 10,992 & 8,071 \\
\hline Germany & 2,124 & 2,344 & 2,968 & 4,563 & 3,997 \\
\hline Latvia & 2,662 & 3,295 & 2,873 & 3,761 & 3,980 \\
\hline Russia & 4,552 & 3,940 & 1,884 & 1,083 & 1,575 \\
\hline Denmark & 1,215 & 1,500 & 1,640 & 1,839 & 2,028 \\
\hline Great Britain & 1,383 & 1,707 & 1,600 & 2,308 & 2,430 \\
\hline Lithuania & 1,449 & 1,543 & 1,174 & 1,487 & 1,725 \\
\hline Netherlands & 1,032 & 823 & 899 & 1,308 & 1,601 \\
\hline Italy & 272 & 303 & 394 & 530 & 563 \\
\hline Other $1 /$ & 5,529 & 6,446 & 6,371 & 10,238 & 12,136 \\
\hline \multirow[t]{2}{*}{ Total } & 31,607 & 37,545 & 35,813 & 55,502 & 57,517 \\
\hline & \multicolumn{5}{|c|}{ (Shares in total exports) } \\
\hline Finland & 19.0 & 22.1 & 22.7 & 31.3 & 33.7 \\
\hline Sweden & 17.1 & 19.5 & 22.0 & 19.8 & 14.0 \\
\hline Germany & 6.7 & 6.2 & 8.3 & 8.2 & 6.9 \\
\hline Latvia & 8.4 & 8.8 & 8.0 & 6.8 & 6.9 \\
\hline Russia & 14.4 & 10.5 & 5.3 & 2.0 & 2.7 \\
\hline Denmark & 3.8 & 4.0 & 4.6 & 3.3 & 3.5 \\
\hline Great Britain & 4.4 & 4.5 & 4.5 & 4.2 & 4.2 \\
\hline Lithuania & 4.6 & 4.1 & 3.3 & 2.7 & 3.0 \\
\hline Netherlands & 3.3 & 2.2 & 2.5 & 2.4 & 2.8 \\
\hline Italy & 0.9 & 0.8 & 1.1 & 1.0 & 1.0 \\
\hline Other $1 /$ & 17.5 & 17.2 & 17.8 & 18.4 & 21.1 \\
\hline Total & 100.0 & 100.0 & 100.0 & 100.0 & 100.0 \\
\hline
\end{tabular}

Source: Bank of Estonia.

1/ Includes exports to "customs-free" zones that were established in November 1999. 
Table 24. Estonia: Direction of Trade - Imports by Countries 1997-2001

\begin{tabular}{|c|c|c|c|c|c|}
\hline & 1997 & 1998 & 1999 & 2000 & 2001 \\
\hline Finland & 18,095 & 20,543 & 18,684 & 26,891 & 22,547 \\
\hline Sweden & 5,169 & 5,572 & 5,042 & 7,638 & 7,519 \\
\hline Germany & 5,099 & 5,751 & 4,775 & 6,841 & 8,397 \\
\hline Russia & 4,153 & 4,173 & 3,933 & 5,880 & 5,842 \\
\hline Latvia & 1,749 & 2,194 & 2,174 & 2,812 & 3,001 \\
\hline Netherlands & 1,888 & 1,941 & 1,636 & 2,205 & 2,919 \\
\hline Denmark & 1,546 & 1,850 & 1,581 & 2,170 & 2,238 \\
\hline Italy & 1,201 & 1,495 & 1,434 & 1,750 & 2,088 \\
\hline Lithuania & 982 & 1,160 & 1,052 & 1,400 & 2,284 \\
\hline Great Britain & 1,207 & 1,375 & 1,026 & 1,407 & 1,546 \\
\hline Other & 7,782 & 9,162 & 9,139 & 13,240 & 16,784 \\
\hline Total & 48,869 & 55,215 & 50,477 & 72,236 & 75,163 \\
\hline Finland & 37.0 & 37.2 & 37.0 & 37.2 & 30.0 \\
\hline Sweden & 10.6 & 10.1 & 10.0 & 10.6 & 10.0 \\
\hline Germany & 10.4 & 10.4 & 9.5 & 9.5 & 11.2 \\
\hline Russia & 8.5 & 7.6 & 7.8 & 8.1 & 7.8 \\
\hline Latvia & 3.6 & 4.0 & 4.3 & 3.9 & 4.0 \\
\hline Netherlands & 3.9 & 3.5 & 3.2 & 3.1 & 3.9 \\
\hline Denmark & 3.2 & 3.4 & 3.1 & 3.0 & 3.0 \\
\hline Italy & 2.5 & 2.7 & 2.8 & 2.4 & 2.8 \\
\hline Lithuania & 2.0 & 2.1 & 2.1 & 1.9 & 3.0 \\
\hline Great Britain & 2.5 & 2.5 & 2.0 & 1.9 & 2.1 \\
\hline Other & 15.9 & 16.6 & 18.1 & 18.3 & 22.3 \\
\hline Total & 100.0 & 100.0 & 100.0 & 100.0 & 100.0 \\
\hline
\end{tabular}

Source: Bank of Estonia. 
Table 25. Estonia: Composition of Trade - Exports by Commodities 1997-2001

\begin{tabular}{|c|c|c|c|c|c|}
\hline & 1997 & 1998 & 1999 & 2000 & 2001 \\
\hline & \multicolumn{5}{|c|}{ (In millions of kroons) } \\
\hline Foodstuffs & 5,121 & 4,969 & 3,176 & 3,779 & 4,629 \\
\hline Mineral products & 1,393 & 977 & 880 & 1,306 & 1,217 \\
\hline Chemical industry products & 2,674 & 2,760 & 2,390 & 3,283 & 3,614 \\
\hline Clothing, footwear, headgear & 5,138 & 6,018 & 5,946 & 7,381 & 8,053 \\
\hline Timber and paper products & 5,221 & 6,424 & 7,065 & 8,747 & 8,758 \\
\hline Metal and metal products & 2,160 & 3,022 & 2,719 & 3,855 & 3,945 \\
\hline Machinery and equipment & 5,725 & 8,335 & 8,478 & 20,208 & 18,948 \\
\hline Transport vehicles & 1,126 & 1,248 & 1,042 & 1,395 & 1,858 \\
\hline Furniture & 1,862 & 2,391 & 2,756 & 3,640 & 4,664 \\
\hline Other goods & 1,187 & 1,400 & 1,361 & 1,907 & 1,832 \\
\hline \multirow[t]{2}{*}{ Total } & 31,607 & 37,545 & 35,813 & 55,502 & 57,518 \\
\hline & \multicolumn{5}{|c|}{ (Shares in total exports) } \\
\hline Foodsuffs & 16.2 & 13.2 & 8.9 & 6.8 & 8.0 \\
\hline Mineral products & 4.4 & 2.6 & 2.5 & 2.4 & 2.1 \\
\hline Chemical industry products & 8.5 & 7.4 & 6.7 & 5.9 & 6.3 \\
\hline Clothing, footwear, headgear & 16.3 & 16.0 & 16.6 & 13.3 & 14.0 \\
\hline Timber and paper products & 16.5 & 17.1 & 19.7 & 15.8 & 15.2 \\
\hline Metal and metal products & 6.8 & 8.0 & 7.6 & 6.9 & 6.9 \\
\hline Machinery and equipment & 18.1 & 22.2 & 23.7 & 36.4 & 32.9 \\
\hline Transport vehicles & 3.6 & 3.3 & 2.9 & 2.5 & 3.2 \\
\hline Furniture & 5.9 & 6.4 & 7.7 & 6.6 & 8.1 \\
\hline Other goods & 3.8 & 3.7 & 3.8 & 3.4 & 3.2 \\
\hline Total & 100.0 & 100.0 & 100.0 & 100.0 & 100.0 \\
\hline
\end{tabular}

Source: Bank of Estonia. 
Table 26. Estonia: Composition of Trade - Imports by Commodities 1997-2001

\begin{tabular}{|c|c|c|c|c|c|}
\hline & 1997 & 1998 & 1999 & 2000 & 2001 \\
\hline & \multicolumn{5}{|c|}{ (In millions of kroons) } \\
\hline Foodstuffs & 6,206 & 6,260 & 5,454 & 6,182 & 7,063 \\
\hline Mineral products & 3,857 & 3,198 & 3,028 & 4,416 & 4,617 \\
\hline Chemical industry products & 5,980 & 6,382 & 6,438 & 8,051 & 8,863 \\
\hline Clothing, footwear, headgear & 5,358 & 6,070 & 5,666 & 6,892 & 7,741 \\
\hline Timber and paper products & 2,301 & 2,729 & 2,642 & 3,508 & 3,929 \\
\hline Metal and metal products & 4,137 & 5,114 & 4,134 & 5,872 & 6,116 \\
\hline Machinery and equipment & 12,349 & 16,262 & 15,525 & 27,789 & 25,189 \\
\hline Transport vehicles & 5,173 & 5,305 & 3,829 & 4,999 & 6,680 \\
\hline Furniture & 1,279 & 1,440 & 1,289 & 1,628 & 1,940 \\
\hline Other goods & 2,228 & 2,454 & 2,471 & 2,898 & 3,024 \\
\hline \multirow[t]{2}{*}{ Total } & 48,869 & 55,215 & 50,477 & 72,236 & 75,163 \\
\hline & \multicolumn{5}{|c|}{ (Shares in total imports) } \\
\hline Foodsuuffs & 12.7 & 11.3 & 10.8 & 8.6 & 9.4 \\
\hline Mineral products & 7.9 & 5.8 & 6.0 & 6.1 & 6.1 \\
\hline Chemical industry products & 12.2 & 11.6 & 12.8 & 11.1 & 11.8 \\
\hline Clothing, footwear, headgear & 11.0 & 11.0 & 11.2 & 9.5 & 10.3 \\
\hline Timber and paper products & 4.7 & 4.9 & 5.2 & 4.9 & 5.2 \\
\hline Metal and metal products & 8.5 & 9.3 & 8.2 & 8.1 & 8.1 \\
\hline Machinery and equipment & 25.3 & 29.5 & 30.8 & 38.5 & 33.5 \\
\hline Transport vehicles & 10.6 & 9.6 & 7.6 & 6.9 & 8.9 \\
\hline Furniture & 2.6 & 2.6 & 2.6 & 2.3 & 2.6 \\
\hline Other goods & 4.6 & 4.4 & 4.9 & 4.0 & 4.0 \\
\hline Total & 100.0 & 100.0 & 100.0 & 100.0 & 100.0 \\
\hline
\end{tabular}

Source: Bank of Estonia. 
Table 27. Estonia: Foreign Direct Investment Inflows by Countries 1997-2001

\begin{tabular}{lrrrrr}
\hline & 1997 & 1998 & 1999 & 2000 & 2001 \\
& & \multicolumn{5}{c}{ (In millions of kroons) } \\
Denmark & 212 & 469 & 309 & 109 & 67 \\
Finland & 1,128 & 1,741 & 1,713 & 2,600 & 2,457 \\
Germany & 183 & 225 & 60 & 195 & 109 \\
Latvia & 5 & -25 & 40 & -6 & 156 \\
Lithuania & -37 & 4 & 105 & -14 & 173 \\
Netherlands & 205 & 46 & -21 & 272 & 1,738 \\
Norway & 747 & 231 & 191 & 52 & -179 \\
Russia & -54 & -193 & 74 & -86 & 58 \\
Sweden & 366 & 4,780 & 1,435 & 2,645 & 2,282 \\
United Kingdom & 188 & 387 & 116 & 117 & 715 \\
USA & 139 & 145 & 400 & 144 & 1,335 \\
Other & 606 & 263 & 26 & 617 & 442 \\
$\quad$ Total & 3,694 & 8,074 & 4,448 & 6,645 & 9,353 \\
Memorandim Items: & \multicolumn{7}{c}{ (In percent of total) } & \\
Finland & 30.5 & 21.6 & 38.5 & 39.1 & 26.3 \\
Sweden & 9.9 & 59.2 & 32.3 & 39.8 & 24.4 \\
USA & 3.8 & 1.8 & 9.0 & 2.2 & 14.3 \\
\hline
\end{tabular}

Source: Bank of Estonia.

Table 28. Estonia: Foreign Direct Investment Outflows by Countries 1997-2001 1/

\begin{tabular}{|c|c|c|c|c|c|}
\hline & 1997 & 1998 & 1999 & 2000 & 2001 \\
\hline & \multicolumn{5}{|c|}{ (In millions of kroons) } \\
\hline Finland & -26 & 42 & 46 & 131 & -6 \\
\hline Lithuania & -480 & -35 & -689 & -398 & $-2,324$ \\
\hline Latvia & -878 & -332 & -694 & -539 & -578 \\
\hline Russia & -102 & 31 & 4 & -97 & 1 \\
\hline Sweden & -1 & -23 & -21 & -3 & -3 \\
\hline Ukraine & -94 & -76 & -3 & 8 & -40 \\
\hline Other & -332 & 312 & 118 & -143 & -291 \\
\hline Total & $-1,913$ & -82 & $-1,240$ & $-1,043$ & $-3,241$ \\
\hline Memorandum Items: & \multicolumn{5}{|c|}{ (In percent of total) } \\
\hline Latvia & 45.9 & 406.7 & 56.0 & 51.7 & 17.8 \\
\hline Lithuania & 25.1 & 43.1 & 55.5 & 38.2 & 71.7 \\
\hline
\end{tabular}

Source: Bank of Estonia.

1/ A negative sign indicates an investment outflow. 
Table 29. Estonia: Foreign Direct Investment Inflows by Sectors 1997-2001

\begin{tabular}{|c|c|c|c|c|c|}
\hline & 1997 & 1998 & 1999 & 2000 & 2001 \\
\hline & \multicolumn{5}{|c|}{ (In millions of kroons) } \\
\hline Agriculture, Fishing, Energy, Gas, Water (inc. Mining) & 80 & 233 & 137 & 66 & -39 \\
\hline Manufacturing & 1,238 & 1,545 & 1,145 & 1,101 & 1,365 \\
\hline Construction & 63 & 161 & 25 & 197 & 209 \\
\hline Trade & 553 & 942 & 443 & 421 & 1,367 \\
\hline Hotels and restaurants & 77 & 37 & 41 & 270 & 77 \\
\hline Transports and communication & 861 & 300 & 1,075 & 1,046 & 1,116 \\
\hline Financial intermediation & 624 & 4,309 & 909 & 1,757 & 2,247 \\
\hline Real estale and business activities & 168 & 424 & 263 & 1,249 & 680 \\
\hline Others & 30 & 123 & 409 & 538 & 2,331 \\
\hline Total & 3,694 & 8,074 & 4,448 & 6,645 & 9,353 \\
\hline Memorandum Items: & \multicolumn{5}{|c|}{ (In percent of total) } \\
\hline Manufacturing & 33.5 & 19.1 & 25.8 & 16.6 & 14.6 \\
\hline Transports and communication & 23.3 & 3.7 & 24.2 & 15.7 & 11.9 \\
\hline Financial intermediation & 16.9 & 53.4 & 20.4 & 26.4 & 24.0 \\
\hline
\end{tabular}

Source: Bank of Estonia.

Table 30. Estonia: Foreign Direct Investment Outflows by Sectors 1997-2001 1/

\begin{tabular}{|c|c|c|c|c|c|}
\hline & 1997 & 1998 & 1999 & 2000 & 2001 \\
\hline & \multicolumn{5}{|c|}{ (In millions of kroons) } \\
\hline Agriculture, Fishing, Energy, Gas, Water (inc. Mining) & 0 & n.a. & n.a. & n.a. & n.a. \\
\hline Manufacturing & -203 & 42 & -327 & -286 & -614 \\
\hline Construction & -14 & 4 & 5 & -21 & -29 \\
\hline Trade & -81 & -179 & -5 & 51 & -33 \\
\hline Hotels and restaurants & n.a. & n.a. & n.a. & -2 & -3 \\
\hline Transports and communication & -325 & 390 & 37 & -76 & -473 \\
\hline Financial intermediation & $-1,108$ & -317 & -866 & 58 & $-1,712$ \\
\hline Real estate and business activities & -176 & -9 & -83 & -750 & -348 \\
\hline Others & -5 & -13 & $-1,240$ & -788 & -30 \\
\hline Total & $-1,913$ & -82 & $-1,240$ & $-1,043$ & $-3,241$ \\
\hline Memorandum Items: & \multicolumn{5}{|c|}{ (In percent of total) } \\
\hline Manufacturing & 10.6 & -51.2 & 26.4 & 27.4 & 19.0 \\
\hline Transports and communication & 17.0 & -475.6 & -3.0 & 7.2 & 14.6 \\
\hline Financial intermediation & 57.9 & 386.6 & 69.9 & -5.5 & 52.8 \\
\hline
\end{tabular}

Source: Bank of Estonia.

$1 /$ n.a. denotes that data are not published due to confidentiality provisions (when there are less than 3 projects during the reporting period). 
Table 31. Estonia: Gross External Debt, 1997-2001 1/

\begin{tabular}{|c|c|c|c|c|c|}
\hline & 1997 & 1998 & 1999 & 2000 & 2001 \\
\hline & \multicolumn{5}{|c|}{ (In millions of euro) } \\
\hline Gross external debt & 2,347 & 2,506 & 2,863 & 3,243 & 3,713 \\
\hline Public & 199 & 201 & 238 & 211 & 192 \\
\hline general govemment & 176 & 200 & 237 & 208 & 191 \\
\hline monetary authorities & 22 & 1 & 1 & 3 & 1 \\
\hline Private & 2,148 & 2,305 & 2,625 & 3,032 & 3,521 \\
\hline trade credits & 230 & 277 & 316 & 394 & 390 \\
\hline bank liabilities & 922 & 831 & 970 & 1,184 & 1,284 \\
\hline other private sector liabilities & 997 & 1,197 & 1,339 & 1,453 & 1,847 \\
\hline Of which: direct investment, intercompany lending & 481 & 510 & 644 & 757 & 989 \\
\hline Short term & 814 & 777 & 965 & 1,183 & 1,246 \\
\hline Public & 0 & 0 & 0 & 3 & 1 \\
\hline Of which: general government & 0 & 0 & 0 & 0 & 0 \\
\hline Private & 814 & 776 & 965 & 1,180 & 1,245 \\
\hline Of which: banks & 475 & 365 & 510 & 664 & 717 \\
\hline Long term & 1,533 & 1,729 & 1,898 & 2,059 & 2,467 \\
\hline Public & 199 & 201 & 238 & 208 & 191 \\
\hline Of which: general government & 176 & 200 & 237 & 208 & 191 \\
\hline Private & 1,334 & 1,529 & 1,661 & 1,851 & 2,276 \\
\hline \multirow[t]{2}{*}{ Of which: banks } & 447 & 466 & 460 & 521 & 567 \\
\hline & & \multicolumn{4}{|c|}{ (In percent of GDP) } \\
\hline Gross external debt & 57.4 & 53.3 & 58.7 & 59.4 & 61.0 \\
\hline Public & 4.9 & 4.3 & 4.9 & 3.9 & 3.2 \\
\hline general government & 4.3 & 4.3 & 4.9 & 3.8 & 3.1 \\
\hline monetary authorities & 0.5 & 0,0 & 0.0 & 0.1 & 0.0 \\
\hline Private & 52.5 & 49.1 & 53.8 & 55.5 & 57.8 \\
\hline trade credits & 5.6 & 5.9 & 6.5 & 7.2 & 6.4 \\
\hline bank liabilities & 22.5 & 17.7 & 19.9 & 21.7 & 21.1 \\
\hline other private sector liabilities & 24.4 & 25.5 & 27.4 & 26.6 & 30.3 \\
\hline Of which: debt liabilities under FDI & 6.0 & 5.5 & 6.7 & 7.1 & 9.3 \\
\hline Short term & 19.9 & 16.5 & 19.8 & 21.7 & 20.5 \\
\hline Public & 0.0 & 0.0 & 0.0 & 0.1 & 0.0 \\
\hline Of which: general government & 0.0 & 0.0 & 0.0 & 0.0 & 0.0 \\
\hline Private & 19.9 & 16.5 & 19.8 & 21.6 & 20.4 \\
\hline Of which: banks & 11.6 & 7.8 & 10.5 & 12.2 & 11.8 \\
\hline Lang term & 37.5 & 36.8 & 38.9 & 37.7 & 40.5 \\
\hline Public & 4.9 & 4.3 & 4.9 & 3.8 & 3.1 \\
\hline Of which: general government & 4.3 & 4.3 & 4.9 & 3.8 & 3.1 \\
\hline Private & 32.6 & 32.5 & 34.0 & 33.9 & 37.4 \\
\hline Of which: banks & 10.9 & 9.9 & 9.4 & 9.5 & 9.3 \\
\hline
\end{tabular}

Source: National authorities and staff calculations.

1/ External debt figures were substantially revised in 2000. 
Table 32. Estonia: Foreign Assets, 1997-2001

\begin{tabular}{|c|c|c|c|c|c|}
\hline & 1997 & 1998 & 1999 & 2000 & 2001 \\
\hline & \multicolumn{5}{|c|}{ (In millions of euro) } \\
\hline Foreign assets & 1,677 & 1,801 & 2,122 & 2,531 & 2,797 \\
\hline Public & 721 & 781 & 981 & 1,087 & 1,049 \\
\hline general government & 25 & 84 & 129 & 94 & 111 \\
\hline monetary authorities & 697 & 697 & 852 & 993 & 938 \\
\hline Private & 955 & 1,020 & 1,142 & 1,444 & 1,749 \\
\hline trade credits & 180 & 245 & 236 & 247 & 317 \\
\hline bank claims & 520 & 424 & 581 & 648 & 848 \\
\hline other private sector claims & 256 & 351 & 324 & 549 & 584 \\
\hline Short term & 1,449 & 1,508 & 1,720 & 2,032 & 3,491 \\
\hline Public & 721 & 781 & 981 & 1,086 & 2,266 \\
\hline Of which: general government & 25 & 84 & 129 & 94 & 1,041 \\
\hline Private & 728 & 727 & 740 & 946 & 1,225 \\
\hline Of which: banks & 350 & 248 & 329 & 410 & 625 \\
\hline Long term & 228 & 294 & 402 & 499 & 531 \\
\hline Public & 0 & 0 & 0 & 1 & 8 \\
\hline Of which: general government & 0 & 0 & 0 & 0 & 0 \\
\hline Private & 228 & 294 & 402 & 497 & 524 \\
\hline \multirow[t]{2}{*}{ Of which: banks } & 170 & 176 & 252 & 238 & 223 \\
\hline & \multicolumn{5}{|c|}{ (In percent of GDP) } \\
\hline Foreign assets & 41.0 & $38.3^{-}$ & 43.5 & 46.4 & 46.0 \\
\hline Public & 17.6 & 16.6 & 20.1 & 19.9 & 17.2 \\
\hline general government & 0.6 & 1.8 & 2.6 & 1.7 & 1.8 \\
\hline monetary authorities & 17.0 & 14.8 & 17.5 & 18.2 & 15.4 \\
\hline Private & 23.3 & 21.7 & 23.4 & 26.4 & 28.7 \\
\hline trade credits & 4.4 & 5.2 & 4.8 & 4.5 & 5.2 \\
\hline bank claims & 12.7 & 9.0 & 11.9 & 11.9 & 13.9 \\
\hline other private sector claims & 6.2 & 7.5 & 6.6 & 10.1 & 9.6 \\
\hline Short term & 35.4 & 32.1 & 35.3 & 37.2 & 57.3 \\
\hline Public & 17.6 & 16.6 & 20.1 & 19.9 & 37.2 \\
\hline Of which: general government & 0.6 & 1.8 & 2.6 & 1.7 & 17.1 \\
\hline Private & 17.8 & 15.5 & 15.2 & 17.3 & 20.1 \\
\hline Of which: banks & 8.6 & 5.3 & 6.7 & 7.5 & 10.3 \\
\hline Long term & 5.6 & 6.2 & 8.2 & 9.1 & 8.7 \\
\hline Public & 0.0 & 0.0 & 0.0 & 0.0 & 0.1 \\
\hline Of which: general government & 0.0 & 0.0 & 0.0 & 0.0 & 0.0 \\
\hline Private & 5.6 & 6.2 & 8.2 & 9.1 & 8.6 \\
\hline Of which banks & 4.2 & 3.8 & 5.2 & 4.4 & $\cdot 3.7$ \\
\hline
\end{tabular}

Source: National authorities and staff calculations. 
Table 33. Estonia: Net External Debt, 1997-2001

\begin{tabular}{|c|c|c|c|c|c|}
\hline & 1997 & 1998 & 1999 & 2000 & 2001 \\
\hline & \multicolumn{5}{|c|}{ (In miltions of euro) } \\
\hline Gross external debt & 2,347 & 2,506 & 2,863 & 3,243 & 3,713 \\
\hline Foreign Assets & 1,677 & 1,801 & 2,122 & 2,531 & 2,797 \\
\hline Net extemal debt & 670 & 705 & 741 & 712 & 915 \\
\hline Public sector & -522 & -580 & -743 & -876 & -857 \\
\hline general government & 152 & 116 & 108 & 114 & 80 \\
\hline monetary authorities & -674 & -696 & -851 & -990 & -937 \\
\hline Private sector & 1,193 & 1,285 & 1,484 & 1,588 & 1,772 \\
\hline Trade credits & 50 & 32 & 80 & 148 & 73 \\
\hline bank liabilicies & 402 & 407 & 389 & 537 & 436 \\
\hline other private sector liabilitics & 741 & 845 & 1,014 & 903 & 1,263 \\
\hline Of which: direct investment, intercompany lending & 481 & 510 & 644 & 757 & 989 \\
\hline Short term & -635 & -731 & -756 & -849 & $\cdot 2,245$ \\
\hline Public & .721 & -781 & -981 & $-1,083$ & $-2,265$ \\
\hline Of which: general government & -25 & -84 & -129 & -94 & $-1,041$ \\
\hline Private & 86 & 50 & 225 & 234 & 20 \\
\hline Of which: banks & 124 & 117 & 181 & 254 & 92 \\
\hline Long term & 1,305 & 1,436 & 1,496 & 1,561 & 1,935 \\
\hline Public & 199 & 201 & 238 & 207 & 183 \\
\hline Of which: general government & 176 & 200 & 237 & 208 & 191 \\
\hline Private & 1,107 & 1,235 & 1,259 & 1,354 & 1,752 \\
\hline \multirow[t]{2}{*}{ Of which: banks } & 277 & 290 & 208 & 283 & 344 \\
\hline & \multicolumn{5}{|c|}{ (In percent of GDP) } \\
\hline Gross external debt & 57.4 & 53.3 & 58.7 & 59.4 & 61.0 \\
\hline Foreign Assets & 41.0 & 38.3 & 43.5 & 46.4 & 46.0 \\
\hline Net external debt & 16.4 & 15.0 & 15.2 & 13.0 & 15.0 \\
\hline Public sector & -12.8 & -12.3 & -15.2 & -16.0 & -14.1 \\
\hline general government & 3.7 & 2.5 & 2.2 & 2.1 & 1.3 \\
\hline Inonetary authorities & -16.5 & -14.8 & -17.5 & -18.1 & -15.4 \\
\hline Private sector & 29.1 & 27.3 & 30.4 & 29.1 & 29.1 \\
\hline Trade credits & 1.2 & 0.7 & 1.6 & 2.7 & 1.2 \\
\hline bank liabilities & 9.8 & 8.7 & 8.0 & 9.8 & 7.2 \\
\hline other private sector liabilities & 18.1 & 18.0 & 20.8 & 16.5 & 20.7 \\
\hline Of which: debt liabilities under FDI & 6.0 & 5.5 & 6.7 & 7.1 & 9.3 \\
\hline Short term & -15.5 & -15.6 & -1.5 .5 & -15.5 & -36.9 \\
\hline Public & -17.6 & -16.6 & -20.1 & -19.8 & -37.2 \\
\hline Of which: general government & -0.6 & -1.8 & -2.6 & -1.7 & -17.1 \\
\hline Private & 2.1 & 1.1 & 4.6 & 4.3 & 0.3 \\
\hline Of which: banks & 3.0 & 2.5 & 3.7 & 4.7 & 1.5 \\
\hline Long term & 31.9 & 30.6 & 30.7 & 28.6 & 31.8 \\
\hline Public & 4.9 & 4.3 & 4.9 & 3.8 & 3.0 \\
\hline Of which: general government & 4.3 & 4.3 & 4.9 & 3.8 & 3.1 \\
\hline Private & 27.0 & 26.3 & 25.8 & 24.8 & 28.8 \\
\hline Of which: banks & 6.8 & 6.2 & 4.3 & 5.2 & 5.7 \\
\hline
\end{tabular}

Source: National authorities and staff calculations based on Table 31 and 32. 